\title{
On an inverse problem of nonlinear imaging with fractional damping
}

\author{
Barbara Kaltenbacher* $\quad$ William Rundell ${ }^{\dagger}$
}

\begin{abstract}
This paper considers the attenuated Westervelt equation in pressure formulation. The attenuation is by various models proposed in the literature and characterised by the inclusion of non-local operators that give power law damping as opposed to the exponential of classical models. The goal is the inverse problem of recovering a spatially dependent coefficient in the equation, the parameter of nonlinearity $\kappa(x)$, in what becomes a nonlinear hyperbolic equation with nonlocal terms. The overposed measured data is a time trace taken on a subset of the domain or its boundary. We shall show injectivity of the linearised map from $\kappa$ to the overposed data used to recover it and from this basis develop and analyse Newton-type schemes for its effective recovery.
\end{abstract}

Keywords: Inverse problems, damped nonlinear wave equation, ultrasound.

Ams classification: 35R30, 35R11, 35K55, 78A46

\section{Introduction}

The problem of nonlinearity parameter imaging with ultrasound in lossy media amounts to identification of the space dependent coefficient $\kappa(x)$ for the attenuated Westervelt equation in pressure formulation

$$
\begin{aligned}
& \left(1-\kappa(x) v^{2}\right)_{t t}-c_{0}^{2} \Delta v+D v=r \text { in } \Omega \times(0, T) \\
& v=0 \text { on } \partial \Omega \times(0, T) ; \quad v(0)=0, \quad v_{t}(0)=0 \text { in } \Omega
\end{aligned}
$$

from observations Here $c_{0}>0$ is the wave speed (possibly space dependent as well), and $D v$ a damping term that will be specified below. For simplicity we impose homogeneous Dirichlet boundary conditions here, but the ideas in this paper extend to more realistic boundary conditions, such as absorbing boundary conditions for avoiding spurious reflections and/or inhomogeneous Neumann boundary conditions for modelling excitation via, e.g., some transducer array. Note that the excitation here is modelled by an interior source $r$, and we refer to a discussion on this in [16].

By letting our $v$ equation satisfy boundary (or possibly interior) observations we obtain an inverse problem for the recovery of $\kappa$. These measurements will be taken to be

$$
g(t)=v\left(x_{0}, t\right) \quad \text { or } \quad g(x, t)=v(x, t), x \in \Sigma, t \in(0, T) .
$$

\footnotetext{
*Department of Mathematics, Alpen-Adria-Universität Klagenfurt. barbara.kaltenbacher@aau.at.

${ }^{\dagger}$ Department of Mathematics, Texas A\&M University, Texas 77843. rundell@math.tamu.edu
} 
at some point $x_{0}$ or on some surface $\Sigma$ contained in $\bar{\Omega}$.

The inverse problem represented by equations (11) and (2) is challenging on at least three counts. First, the underlying model equation is nonlinear and in fact the nonlinearity occurs in the highest order term. Second, the unknown coefficient $\kappa(x)$ is directly coupled to this term and third, is spatially varying whereas the data $g(t)$ is in the "orthogonal" time direction and this is well known to lead to several ill-conditioning of the inversion of the map from data to unknown.

The use of ultrasound is a well-established protocol in the imaging of human tissue and, besides the classical sonography methodology, there exist several novel imaging principle, such as harmonic imaging or nonlinearity imaging. The latter [2, 3, 5, 13, 24, 26, 27], relies on tissue-dependence, hence spatial variation of the parameter of nonlinearity $B / A$ that is contained in $\kappa$. See, e.g., It thus inherently needs a nonlinear acoustic model as an underlying PDE and we refer to e.g., the review [15] and the references therein for a brief derivation of the fundamental nonlinear acoustic equations. The quantity of interest from an imaging perspective is the coefficient $\kappa$ and its recovery in the case when the damping term was $D u=b \triangle u_{t}$ was the subject of [16]. This is the classical formulation of damping being proportional to velocity but there are may alternative models that are prominent in the literature. We mention some of these in the next section but the main change is the incorporation of non-local terms involving either fractional derivatives in time or modifying the operator $(-\triangle)$ to have the Laplacian raised to a fractional power $(-\triangle)^{\beta}$. These have the effect of ameliorating the exponential decay of the solution, by a fractional exponent in the frequencies in the case of $(-\triangle)^{\beta}$ and by a power law decay in the case of a fractional time derivative. The use of such operators in inverse problems is now well documented in the literature (see, [14] and in particular, for the wave equation in [17]).

In this paper we will provide analysis for the forwards problem and in particular regularity and well-posedness for the coefficient-to-state map $G: \kappa \mapsto v$ where $v$ solves (1). The forwards map is defined by $F(\kappa)=\operatorname{tr}_{\Sigma} v$, where $\operatorname{tr}_{\Sigma} v$ denotes the time trace of the spaceand-time dependent function $v:(0, T) \times \Omega$ at the observation surface $\Sigma$ (which may also just be a single point $\Sigma=\left\{x_{0}\right\}$ ). Its linearization at $\kappa=0$ is $F^{\prime}(0)$ and we will prove an injectivity result which will both show local uniqueness and pave the way for the use of Newton's method which we will analyse in section 4 then show reconstructions based on this approach in section 6 ,

\section{The Imaging Problem}

As already mentioned in the introduction, the inverse problem under consideration is to recover the space dependent coefficient $\kappa(x)$ in the attenuated Westervelt equation which can also be written in the form

$$
\begin{aligned}
& u_{t t}+c^{2} \mathscr{A} u+D u=\kappa(x)\left(u^{2}\right)_{t t}+r \text { in } \Omega \times(0, T) \\
& u(0)=0, \quad u_{t}(0)=0 \text { in } \Omega
\end{aligned}
$$

from observations $g(x, t)=u(x, t), x \in \Sigma, t \in(0, T)$ where $\Sigma$ may be a single point, typically located on $\partial \Omega$. Here $c>0$ is the constant mean wave speed, and $\mathscr{A}=-\left(c_{0}(x)^{2} / c^{2}\right) \triangle$ contains the possibly spatially varying coefficient $c_{0}(x)>0$ and is equipped - for simplicity - with homogeneous boundary conditions. Moreover $r=r(x, t)$ is a known source term 
modelling excitation of the acoustic wave by a transducer array, see [17].

The damping operator $D$ appearing in (3) is a differential operator containing space and/or time derivatives. Classically, $D$ will consist of integer derivatives, typical examples being $D=\mathscr{A} \partial_{t}$ or $D=\partial_{t}$ often referred to as strong and weak damping, respectively. We here list some of the (due to experimentally found power law frequency dependence) practically relevant fractional damping models, that we have already discussed in [17] in a different imaging context, namely for the inverse PAT/TAT problem:

\section{Time fractional models:}

- Caputo-Wismer model [6], [25, eq. (5)], called Kelvin wave equation in [4, eq. (19)]

$$
D=b \mathscr{A} \partial_{t}^{\alpha}
$$

where typically $\alpha \in[0,1]$.

- (Modified) Szabo model [22], [4, eq. (42)]

$$
D=b \partial_{t}^{\alpha+2}
$$

where $\alpha \in[-1,1], b \geq 0$.

- Fractional Zener (combined Caputo-Wismer-Szabo) model [12, 18], [4, eq. (30)]

$$
D=b_{1} \mathscr{A} \partial_{t}^{\alpha_{1}}+b_{2} \partial_{t}^{\alpha_{2}+2}
$$

where $\alpha_{1} \geq \alpha_{2} \in[0,1], b_{1} \geq b_{2} c^{2}$, cf. [12, Section III.B].

In these models $\partial_{t}^{\alpha}$ denotes the Djrbashian-Caputo fractional time derivative, which here, due to the homogeneous initial conditions, coincides with the Riemann-Liouville one.

\section{Space fractional models:}

- Chen-Holm model [7, eq. (21)]

$$
D=b \mathscr{A}^{\beta}
$$

typically with $\beta \in[0,1]$, where Kelvin-Voigt damping is recovered when $\beta=1$.

- Treeby-Cox model [23, eq. (28)]

$$
D=b_{1} \mathscr{A}^{\beta} \partial_{t}+b_{2} \mathscr{A}^{\beta+1 / 2}
$$

typically $\beta \in[0,1]$, which is an extension of the former.

Here we use the spectral definition of the Laplacian which coincides with the Riesz version on $\mathbb{R}^{d}$; however, they differ in case of bounded $\Omega$.

In this paper, we will focus on two damping models namely (a) a combination of (4) and (7), since we find it interesting to investigate the interplay of space- and time-fractional derivatives and its influence on the ill-posedness of the inverse problem; (b) (6) as it contains higher than second order time derivatives which are in case $\alpha_{2}=1$ known to make the equation behave wave-like (finite speed of propagation) in spite of the damping, which is expected to influence the degree of ill-posedness of the inverse problem as well. Thus we here focus on the two damping models

$$
\begin{aligned}
& D=b \mathscr{A}^{\beta} \partial_{t}^{\alpha} \quad \text { (combination of Caputo-Wismer and Chen-Holm model - CWCH) } \\
& D=b_{1} \mathscr{A} \partial_{t}^{\alpha_{1}}+b_{2} \partial_{t}^{\alpha_{2}+2} \quad \text { (fractional Zener - FZ). }
\end{aligned}
$$




\section{Analysis of the forward problem}

In this section, we consider well-posedness of the initial value problem for the parameterto-state map $G: \kappa \mapsto u$ where $u$ solves (3) and its linearisation $z=G^{\prime}(\kappa) \underline{d \kappa}$

$$
\begin{aligned}
(1-2 \kappa u) z_{t t}+c^{2} \mathscr{A} z+D z-4 \kappa u_{t} z_{t}-2 \kappa u_{t t} z & =2 \underline{\delta \kappa}\left(u u_{t t}+u_{t}^{2}\right) \text { in } \Omega \times(0, T) \\
z(0)=0, \quad z_{t}(0) & =0 \text { in } \Omega
\end{aligned}
$$

for given $\kappa$ and $\underline{\delta \kappa}$, respectively, in the context of several damping models $D$. In order to prove Fréchet differentiability, we will also have to consider the difference $v=G(\tilde{\kappa})-$ $G(\kappa)=\tilde{u}-u$, which solves

$$
\begin{aligned}
(1-2 \kappa u) v_{t t} & +c^{2} \mathscr{A} v+D v-2 \kappa\left(\tilde{u}_{t}+u_{t}\right) v_{t}-2 \kappa \tilde{u}_{t t} v \\
& =2(\tilde{\kappa}-\kappa)\left(\tilde{u} \tilde{u}_{t t}+\tilde{u}_{t}^{2}\right) \text { in } \Omega \times(0, T) \\
v(0)=0, \quad v_{t}(0) & =0 \text { in } \Omega
\end{aligned}
$$

as well as the first order Taylor remainder $w=G(\tilde{\kappa})-G(\kappa)-G^{\prime}(\kappa)(\tilde{\kappa}-\kappa)$ which satisfies

$$
\begin{aligned}
(1-2 \kappa u) w_{t t} & +c^{2} \mathscr{A} w+D w-4 \kappa u_{t} w_{t}-2 \kappa u_{t t} w \\
& =2 \underline{d \kappa}\left(v \tilde{u}_{t t}+u v_{t t}+\left(\tilde{u}_{t}+u_{t}\right) v_{t}\right)+2 \kappa\left(v v_{t t}+v_{t}^{2}\right) \text { in } \Omega \times(0, T) \\
w(0)=0, \quad w_{t}(0) & =0 \text { in } \Omega,
\end{aligned}
$$

Here we can allow for spatially varying sound speed $c_{0}(x)$ for which we only require

$$
c_{0} \in L^{\infty}(\Omega) \text { and } c_{0}(x) \geq c>0
$$

unless otherwise stated, by setting

$$
\mathscr{A}=-\frac{c_{0}^{2}}{c^{2}} \triangle
$$

where $-\triangle$ is the Laplace operator equipped with homogeneous Dirichlet boundary conditions. We denote by $\left(\phi_{j}, \lambda_{j}\right)$ an eigensystem of the operator $\mathscr{A}$ with domain $\dot{H}^{2}(\Omega):=$ $\mathscr{D}(\mathscr{A})$ which is selfadjoint and positive definite with respect to the weighted $L^{2}$ space $\dot{L}^{2}(\Omega):=L_{c^{2} / c_{0}^{2}}^{2}(\Omega)$. Note that we assume $\mathscr{A}^{-1}: \dot{L}^{2}(\Omega) \rightarrow \dot{L}^{2}(\Omega)$ to be compact (which is e.g., satisfied if $\Omega$ is bounded; for some comments on more general domain and boundary settings we point to [17]), so that the eigensystem exists and is complete with $\lambda_{j} \rightarrow \infty$ as $j \rightarrow \infty$. Moreover, this defines a scale of Hilbert spaces $\dot{H}^{s}(\Omega):=\mathscr{D}\left(\mathscr{A}^{s / 2}\right), s \in \mathbb{R}$, whose norm can be defined via the eigensystem as $\|v\|_{\dot{H}^{s}(\Omega)}=\left(\sum_{j=1}^{\infty} \lambda_{j}^{s}\left|\left\langle v, \phi_{j}\right\rangle\right|^{2}\right)^{1 / 2}$. We will denote by $\langle\cdot, \cdot\rangle$ the $\dot{L}^{2}$ inner product (that is, the weighted one) on $\Omega$ whereas the use of the ordinary $L^{2}$ inner product will be indicated by a subscript $\langle\cdot, \cdot\rangle_{L^{2}}$ Moreover, we use the abbreviations $\|u\|_{L_{t}^{p}\left(L^{q}\right)}=\|u\|_{L^{p}\left(0, t ; L^{q}(\Omega)\right)},\|u\|_{L^{p}\left(L^{q}\right)}=\|u\|_{L^{p}\left(0, T ; L^{q}(\Omega)\right)}$ for space-time norms.

Throughout this paper, we denote by $\partial_{t}^{\alpha}$ the (partial) Caputo-Djrbashian fractional time derivative of order $\alpha \in(n-1, n)$ with $n \in \mathbb{N}$ by $\partial_{t}^{\alpha} u=I_{t}^{n-\alpha}\left[\partial_{t}^{n} u\right]$, where $\partial_{t}^{n}$ denotes the $n$-th integer order partial time derivative and for $\gamma \in(0,1)$, and $I_{t}^{\gamma}$ is the Abel integral operator defined by

$$
I_{t}^{\gamma}[v](t)=\frac{1}{\Gamma(\gamma)} \int_{0}^{t} \frac{v(s)}{(t-s)^{1-\gamma}} d s .
$$

For details on fractional differentiation and subdiffusion equations, we refer to, e.g., [8, 9. 19, 20, 21]. See also the tutorial paper on inverse problems for anomalous diffusion 
processes [14]. Whenever we use the Riemann-Liouville fractional derivative $\partial_{t}^{n} I_{t}^{n-\alpha}$, this will be denoted by ${ }^{R L} \partial_{t}^{\alpha}$. These two versions of the fractional derivative coincide when applied to a function whose Cauchy data up to order $n-1$ at $t=0$ vanish.

The crucial tool we need in obtaining the required estimates is the following consequence of Alikhanov's Lemma [1, Lemma 1]

$$
\partial_{t}^{\gamma}[w](t) w(t) \geq \frac{1}{2}\left(\partial_{t}^{\gamma} w^{2}\right)(t)
$$

for any absolutely continuous function $w$. We apply it to $w=\partial_{t}^{\alpha} v$ with $\gamma=1-\alpha$, using the identities

$$
\begin{aligned}
& \partial_{t}^{1-\alpha} w=\partial_{t}^{1-\alpha} \partial_{t}^{\alpha} v=\partial_{t}^{1-\alpha} I_{t}^{1-\alpha} v_{t}=v_{t}, \\
& \int_{0}^{t}\left(\partial_{t}^{1-\alpha} w^{2}\right)(s) d s=\int_{0}^{t} R L \partial_{t}^{1-\alpha}\left[w^{2}\right](s) d s=\int_{0}^{t} \partial_{t} I_{t}^{\alpha}\left[w^{2}\right](s) d s=I_{t}^{\alpha}\left[w^{2}\right](t)
\end{aligned}
$$

that hold for $v_{t} \in L^{\infty}(0, T)$ (Theorem 2.3 in fde-lect.pdf) and for $w^{2} \in W^{1,1}(0, T)$ with $w(0)=0$ (Theorem 2.2 in fde-lect.pdf). Note that for $w=\partial_{t}^{\alpha} v$ we automatically have $w(0)=0$ and $I_{t}^{\alpha}\left[w^{2}\right](t)=0$. After integration with respect to time this implies the following result.

Lemma 3.1. For $v \in W^{1, \infty}(0, T)$ with $\left(\partial_{t}^{\alpha} v\right)^{2} \in W^{1,1}(0, T)$, and $t \in(0, T)$, the following estimate holds.

$$
\int_{0}^{t} \partial_{t}^{\alpha}[v](s) v_{t}(s) d s \geq I_{t}^{\alpha}\left[\left(\partial_{t}^{\alpha} v\right)^{2}\right] \geq \frac{1}{2 \Gamma(\alpha) t^{1-\alpha}}\left\|\partial_{t}^{\alpha} v\right\|_{L^{2}(0, t)}^{2} .
$$

\subsection{Caputo-Wismer-Kelvin damping}

We start with the Caputo-Wismer-Kelvin model

$$
D=b \mathscr{A}^{\beta} \partial_{t}^{\alpha} \quad \text { with } \beta \in[0,1], b \geq 0
$$

and first of all consider the initial boundary value problem for the general linear PDE

$$
\begin{gathered}
(1-\sigma) u_{t t}+c^{2} \mathscr{A} u+b \mathscr{A}^{\beta} \partial_{t}^{\alpha} u+\mu u_{t}+\rho u=h \\
u(0)=u_{0}, \quad u_{t}(0)=u_{1}
\end{gathered}
$$

with constants $b, c>0$ and given space- and time dependent functions $\sigma, \mu, \rho, h$ where $\sigma$ satisfies the non-degeneracy condition

$$
\sigma(x, t) \leq \bar{\sigma}<1 \text { for all } x \in \Omega \quad t \in(0, T) .
$$

In order to prove existence and uniqueness of solutions to (16), (17), we apply the usual Faedo-Galerkin approach of discretisation in space with eigenfunctions of $\mathscr{A}, u(x, t) \approx$ $u^{n}(x, t)=\sum_{i=1}^{n} u_{i}^{n}(t) \phi_{i}(x)$ and testing with $\phi_{j}$, that is,

$$
\left\langle(1-\sigma) u_{t t}^{n}+c^{2} \mathscr{A} u^{n}+b \mathscr{A}^{\beta} \partial_{t}^{\alpha} u^{n}+\mu u_{t}^{n}+\rho u^{n}-h, v\right\rangle=0 \quad v \in \operatorname{span}\left(\phi_{1}, \ldots, \phi_{n}\right) .
$$

This leads to the ODE system

$$
\left(I-S^{n}(t)\right) \underline{u}^{n \prime \prime}(t)+b\left(\Lambda^{n}\right)^{\beta}\left(\partial_{t}^{\alpha} \underline{u}^{n}\right)(t)+M^{n}(t) \underline{u}^{n \prime}(t)+\left(c^{2} \Lambda^{n}+R^{n}(t)\right) \underline{u}^{n}(t)=\underline{h}^{n}
$$

with matrices and vectors defined by

$$
\begin{aligned}
& \underline{u}^{n}(t)=\left(u_{i}^{n}(t)\right)_{i=1, \ldots n}, \quad \underline{h}^{n}(t)=\left(\left\langle h(t), \phi_{i}\right\rangle\right)_{i=1, \ldots n}, \quad \Lambda^{n}=\operatorname{diag}\left(\lambda_{1}, \ldots, \lambda_{n}\right), \\
& S^{n}(t)=\left(\left\langle\sigma(t) \phi_{i}, \phi_{j}\right\rangle\right)_{i, j=1, \ldots n}, \quad M^{n}(t)=\left(\left\langle\mu(t) \phi_{i}, \phi_{j}\right\rangle\right)_{i, j=1, \ldots n}, \\
& R^{n}(t)=\left(\left\langle\rho(t) \phi_{i}, \phi_{j}\right\rangle\right)_{i, j=1, \ldots n} .
\end{aligned}
$$


Existence of a unique solution $\underline{u}^{n} \in C^{2}\left(0, T ; \mathbb{R}^{n}\right)$ to (20) follows from standard oDE theory (Picard-Lindelöf Theorem and Gronwall's Inequality), as long as $\sigma, \mu$ and $\rho$ are in $C\left(0, T ; \dot{H}^{s}(\Omega)\right.$ ) for some $s \in \mathbb{R}$ (noting that the eigenfunctions $\phi_{j}$ are contained in $\dot{H}^{k}(\Omega)$ for any $k \in \mathbb{N}$ and therefore the vector and matrix functions $\underline{h}^{n}, S^{n}, M^{n}, R^{n}$ in (21) are well-defined and contained in $C\left(0, T ; \mathbb{R}^{n}\right)$ and $C\left(0, T ; \mathbb{R}^{n \times n}\right)$, respectively. Moreover due to (18), the symmetric matrix $S^{n}(t)$ is positive definite with smallest eigenvalue bounded away from zero by $1-\bar{\sigma}$ cf (18).

We multiply (20) with $\left(\Lambda^{n}\right)^{2} \underline{u}^{n \prime}(t)$ and integrate with respect to time, using the identity

$$
\begin{aligned}
& \left(S^{n}(t) \underline{u}^{n \prime \prime}(t)\right)^{T}\left(\Lambda^{n}\right)^{2} \underline{u}^{n \prime}(t)=\sum_{i=1}^{n} \sum_{j=1}^{n}\left\langle\sigma(t) \phi_{i}, \phi_{j}\right\rangle \lambda_{j}^{2} u_{i}^{n \prime \prime}(t) u_{j}^{n \prime}(t) \\
& =\sum_{i=1}^{n} \sum_{j=1}^{n}\left\langle\sigma(t) \phi_{i}, \phi_{j}\right\rangle \lambda_{i} u_{i}^{n \prime \prime}(t) \lambda_{j} u_{j}^{n \prime}(t)+\sum_{i=1}^{n} \sum_{j=1}^{n}\left\langle\sigma(t) \phi_{i}, \phi_{j}\right\rangle\left(\lambda_{j}-\lambda_{i}\right) u_{i}^{n \prime \prime}(t) \lambda_{j} u_{j}^{n \prime}(t) \\
& =\frac{1}{2} \frac{d}{d t} \sum_{i=1}^{n} \sum_{j=1}^{n}\left\langle\sigma(t) \phi_{i}, \phi_{j}\right\rangle \lambda_{i} u_{i}^{n \prime}(t) \lambda_{j} u_{j}^{n \prime}(t)-\frac{1}{2} \sum_{i=1}^{n} \sum_{j=1}^{n}\left\langle\sigma_{t}(t) \phi_{i}, \phi_{j}\right\rangle \lambda_{i} u_{i}^{n \prime}(t) \lambda_{j} u_{j}^{n \prime}(t) \\
& \quad+\sum_{i=1}^{n} \sum_{j=1}^{n}\left(\left\langle\sigma(t) \phi_{i}, \mathscr{A} \phi_{j}\right\rangle-\left\langle\sigma(t) \phi_{j}, \mathscr{A} \phi_{i}\right\rangle\right) u_{i}^{n \prime \prime}(t) \lambda_{j} u_{j}^{n \prime}(t)
\end{aligned}
$$

where

$$
\begin{aligned}
& \left\langle\sigma(t) \phi_{i}, \mathscr{A} \phi_{j}\right\rangle-\left\langle\sigma(t) \phi_{j}, \mathscr{A} \phi_{i}\right\rangle=\left\langle\mathscr{A}\left[\sigma(t) \phi_{i}\right]-\sigma(t) \mathscr{A} \phi_{i}, \phi_{j}\right\rangle \\
& =\left\langle-\triangle\left[\sigma(t) \phi_{i}\right]+\sigma(t) \triangle \phi_{i}, \phi_{j}\right\rangle_{L^{2}}=-\left\langle\left(c_{0}^{2} / c^{2}\right)\left(\triangle \sigma(t) \phi_{i}+\nabla \sigma(t) \cdot \nabla \phi_{i}\right), \phi_{j}\right\rangle
\end{aligned}
$$

provided $\sigma(t) \phi_{i} \in \dot{H}^{s}(\Omega)$ ) for some $s \in \mathbb{R}$. (Note that the latter identity also holds true in case of spatially varying $c_{0}$ since we use the $c^{2} / c_{0}^{2}$ weighted $L^{2}$ inner product then). Thus we have

$$
\begin{aligned}
& \int_{0}^{t}\left(\left(I-S^{n}(t)\right) \underline{u}^{n \prime \prime}(s)\right)^{T}\left(\Lambda^{n}\right)^{2} \underline{u}^{n \prime}(s) d s \\
& =\int_{0}^{t}\left(\frac{1}{2} \frac{d}{d t}\left\|\sqrt{1-\sigma} \mathscr{A} u_{t}^{n}\right\|_{\dot{L}^{2}(\Omega)}^{2}(t)+\frac{1}{2}\left\langle\sigma_{t}(t) \mathscr{A} u_{t}^{n}(t), \mathscr{A} u_{t}^{n}(t)\right\rangle\right. \\
& \left.\quad+\left\langle\left(c_{0}^{2} / c^{2}\right)\left(\triangle \sigma(t) u_{t t}^{n}(t)+\nabla \sigma(t) \cdot \nabla u_{t t}^{n}(t)\right), \mathscr{A} u_{t}^{n}(t)\right\rangle\right) \\
& =\frac{1}{2}\left\|\sqrt{1-\sigma(t)} \mathscr{A} u_{t}^{n}(t)\right\|_{\dot{L}^{2}(\Omega)}^{2}-\frac{1}{2}\left\|\sqrt{1-\sigma(0)} \mathscr{A} u_{t}^{n}(0)\right\|_{\dot{L}^{2}(\Omega)}^{2} \\
& \left.\quad+\int_{0}^{t}\left\langle\frac{1}{2} \sigma_{t}(s) \mathscr{A} u_{t}^{n}(s)+\left(c_{0}^{2} / c^{2}\right)\left(\triangle \sigma(s) u_{t t}^{n}(s)+\nabla \sigma(s) \cdot \nabla u_{t t}^{n}(s)\right), \mathscr{A} u_{t}^{n}(s)\right\rangle d s\right) .
\end{aligned}
$$

Similarly, we have

$$
\begin{aligned}
& \left(M^{n}(t) \underline{u}^{n \prime}(t)\right)^{T}\left(\Lambda^{n}\right)^{2} \underline{u}^{n \prime}(t) \\
& =\left\langle\mu(t) \mathscr{A} u_{t}^{n}(t), \mathscr{A} u_{t}^{n}(t)\right\rangle-\left\langle\left(c_{0}^{2} / c^{2}\right)\left(\triangle \mu(t) u_{t}^{n}(t)+\nabla \mu(t) \cdot \nabla u_{t}^{n}(t)\right), \mathscr{A} u_{t}^{n}(t)\right\rangle
\end{aligned}
$$

and

$$
\begin{aligned}
& \left(R^{n}(t) \underline{u}^{n}(t)\right)^{T}\left(\Lambda^{n}\right)^{2} \underline{u}^{n \prime}(t) \\
& =\left\langle\rho(t) \mathscr{A} u^{n}(t), \mathscr{A} u_{t}^{n}(t)\right\rangle-\left\langle\left(c_{0}^{2} / c^{2}\right)\left(\triangle \rho(t) u^{n}(t)+\nabla \rho(t) \cdot \nabla u^{n}(t)\right), \mathscr{A} u_{t}^{n}(t)\right\rangle .
\end{aligned}
$$


Finally,

$$
\begin{aligned}
& \int_{0}^{t}\left(\left(\Lambda^{n}\right)^{\beta}\left(\partial_{t}^{\alpha} \underline{u}^{n}\right)(t)\right)^{T}\left(\Lambda^{n}\right)^{2} \underline{u}^{n \prime}(t)=\int_{0}^{t} \sum_{j=1}^{n} \lambda_{j}^{2+\beta} \int_{0}^{t}\left(\partial_{t}^{\alpha} u_{j}^{n}\right)(s) u_{j}^{n \prime}(s) d s \\
& \geq \frac{1}{2 \Gamma(\alpha) t^{1-\alpha}} \sum_{j=1}^{n} \lambda_{j}^{2+\beta} \int_{0}^{t}\left(\partial_{t}^{\alpha} u_{j}^{n}(s)\right)^{2} d s=\frac{1}{2 \Gamma(\alpha) t^{1-\alpha}}\left\|\mathscr{A}^{1+\beta / 2} \partial_{t}^{\alpha} u^{n}\right\|_{L_{t}^{2}\left(\dot{L}^{2}\right)}^{2}
\end{aligned}
$$

and

$$
\begin{aligned}
& \int_{0}^{t}\left(\Lambda^{n} \underline{u}^{n}(t)\right)^{T}\left(\Lambda^{n}\right)^{2} \underline{u}^{n \prime}(t)=\sum_{j=1}^{n} \lambda_{j}^{3} \int_{0}^{t} u_{j}^{n}(s) u_{j}^{n \prime}(s) d s \\
& =\frac{1}{2} \sum_{j=1}^{n} \lambda_{j}^{3} \int_{0}^{t} \frac{d}{d t}\left(u_{j}^{n}\right)^{2}(s) d s=\frac{1}{2}\left\|\mathscr{A}^{3 / 2} u^{n}(t)\right\|_{\dot{L}^{2}(\Omega)}^{2}-\frac{1}{2}\left\|\mathscr{A}^{3 / 2} u^{n}(0)\right\|_{\dot{L}^{2}(\Omega)}^{2} .
\end{aligned}
$$

This together with Young's inequality yields the energy estimate

$$
\begin{aligned}
& \frac{1}{2}\left\|\sqrt{1-\sigma(t)} \mathscr{A} u_{t}^{n}(t)\right\|_{\dot{L}^{2}(\Omega)}^{2}+\frac{b}{2 \Gamma(\alpha) t^{1-\alpha}}\left\|\mathscr{A}^{1+\beta / 2} \partial_{t}^{\alpha} u^{n}\right\|_{L_{t}^{2}\left(\dot{L}^{2}\right)}^{2}+\frac{c^{2}}{2}\left\|\mathscr{A}^{3 / 2} u^{n}(t)\right\|_{\dot{L}^{2}(\Omega)}^{2} \\
& \leq \frac{1}{2}\left\|\sqrt{1-\sigma(0)} \mathscr{A} u_{t}^{n}(0)\right\|_{\dot{L}^{2}(\Omega)}^{2}+\frac{c^{2}}{2}\left\|\mathscr{A}^{3 / 2} u^{n}(0)\right\|_{\dot{L}^{2}(\Omega)}^{2}+\frac{1}{2 \varepsilon}\left\|\mathscr{A} u_{t}^{n}\right\|_{L_{t}^{2}\left(\dot{L}^{2}\right)}^{2} \\
& +\frac{\varepsilon}{2} \|-\frac{1}{2} \sigma_{t} \mathscr{A} u_{t}^{n}-\mu \mathscr{A} u_{t}^{n}-\rho \mathscr{A} u^{n}+\mathscr{A} h \\
& +\left(c_{0}^{2} / c^{2}\right)\left(-\triangle \sigma u_{t t}^{n}-\nabla \sigma \cdot \nabla u_{t t}^{n}+\triangle \mu u_{t}^{n}+\nabla \mu \cdot \nabla u_{t}^{n}+\triangle \rho u^{n}+\nabla \rho \cdot \nabla u^{n}\right) \|_{L_{t}^{2}\left(\dot{L}^{2}\right)}^{2} \\
& \leq \frac{1}{2}\left\|\sqrt{1-\sigma(0)} \mathscr{A} u_{t}^{n}(0)\right\|_{\dot{L}^{2}(\Omega)}^{2}+\frac{c^{2}}{2}\left\|\mathscr{A}^{3 / 2} u^{n}(0)\right\|_{\dot{L}^{2}(\Omega)}^{2}+\frac{1}{2 \varepsilon}\left\|\mathscr{A} u_{t}^{n}\right\|_{L_{t}^{2}\left(\dot{L}^{2}\right)}^{2} \\
& +\frac{\varepsilon}{2}\left(\frac{1}{2}\left\|\sigma_{t}\right\|_{L_{t}^{2}\left(L^{\infty}\right)}\left\|\mathscr{A} u_{t}^{n}\right\|_{L_{t}^{\infty}\left(L^{2}\right)}+\|\mu\|_{L_{t}^{2}\left(L^{\infty}\right)}\left\|\mathscr{A} u_{t}^{n}\right\|_{L_{t}^{\infty}\left(L^{2}\right)}\right. \\
& +\|\rho\|_{L_{t}^{2}\left(L^{\infty}\right)}\left\|\mathscr{A} u^{n}\right\|_{L_{t}^{\infty}\left(L^{2}\right)}+\|\mathscr{A} h\|_{L_{t}^{2}\left(L^{2}\right)} \\
& +\frac{\left\|c_{0}\right\|_{L^{\infty}(\Omega)}}{c}\left(\|\triangle \sigma\|_{L^{\infty}\left(L^{4}\right)}\left\|u_{t t}^{n}\right\|_{L^{2}\left(L^{4}\right)}+\|\nabla \sigma\|_{L_{t}^{\infty}\left(L^{\infty}\right)}\left\|\nabla u_{t t}^{n}\right\|_{L_{t}^{2}\left(L^{2}\right)}\right. \\
& +\|\triangle \mu\|_{L_{t}^{2}\left(L^{2}\right)}\left\|u_{t}^{n}\right\|_{L_{t}^{\infty}\left(L^{\infty}\right)}+\|\nabla \mu\|_{L^{2}\left(L^{4}\right)}\left\|\nabla u_{t}^{n}\right\|_{L^{\infty}\left(L^{4}\right)} \\
& \left.\left.+\|\triangle \rho\|_{L_{t}^{2}\left(L^{2}\right)}\left\|u^{n}\right\|_{L_{t}^{\infty}\left(L^{\infty}\right)}+\|\nabla \rho\|_{L^{2}\left(L^{4}\right)}\left\|\nabla u^{n}\right\|_{L^{\infty}\left(L^{4}\right)}\right)\right)^{2}
\end{aligned}
$$

Here we can make use of the fact that $\left\|u^{n}\right\|_{L^{\infty}(0, t ; Z)} \leq\left\|u^{n}(0)\right\|_{Z}+\sqrt{T}\left\|u_{t}^{n}\right\|_{L^{2}(0, t ; Z)}$ and the embedding estimates

$$
\begin{aligned}
& \|v\|_{L^{4}(\Omega)} \leq C_{H^{1}, L^{4}}\|\nabla v\|, \quad v \in H_{0}^{1}(\Omega), \\
& \|v\|_{L^{\infty}(\Omega)} \leq C_{H^{2}, L^{\infty}}\|\mathscr{A} v\|_{\dot{L}^{2}(\Omega)}, \quad v \in H_{0}^{1}(\Omega) \cap H^{2}(\Omega),
\end{aligned}
$$

in order to further estimate

$$
\begin{aligned}
\left\|u_{t t}^{n}\right\|_{L^{2}\left(L^{4}\right)} & \leq C_{H^{1}, L^{4}}\left\|\nabla u_{t t}^{n}\right\|_{L_{t}^{2}\left(L^{2}\right)} \\
\left\|u_{t}^{n}\right\|_{L_{t}^{\infty}\left(L^{\infty}\right)} & \leq C_{H^{2}, L^{\infty}}\left\|\mathscr{A} u_{t}^{n}\right\|_{L_{t}^{\infty}\left(L^{2}\right)} \\
\left\|u^{n}\right\|_{L_{t}^{\infty}\left(L^{\infty}\right)} & \leq C_{H^{2}, L^{\infty}}\left\|\mathscr{A} u^{n}\right\|_{L_{t}^{\infty}\left(L^{2}\right)} \\
\left\|\nabla u^{n}\right\|_{L_{t}^{\infty}\left(L^{\infty}\right)} & \leq C_{H^{2}, L^{\infty}}\left\|\mathscr{A}^{3 / 2} u^{n}\right\|_{L_{t}^{\infty}\left(L^{2}\right)}
\end{aligned}
$$

Now we proceed with estimating $\left\|\nabla u_{t t}^{n}\right\|_{L^{2}}^{2}$ by multiplying the ODE (20) with $\Lambda^{n} \underline{u}^{n \prime \prime}$, that is testing (19) with $v=\mathscr{A} u_{t t}^{n}(t)$, and using integration by parts (note that all terms in $(1-$ 
$\sigma) u_{t t}^{n}+c^{2} \mathscr{A} u^{n}+b \mathscr{A}^{\beta} \partial_{t}^{\alpha} u^{n}+\mu u_{t}^{n}+\rho u^{n}-h$ vanish on $\left.\partial \Omega\right)$ as well as Young's inequality

$$
\begin{aligned}
& 0=\left\langle(1-\sigma) u_{t t}^{n}+c^{2} \mathscr{A} u^{n}+b \mathscr{A}^{\beta} \partial_{t}^{\alpha} u^{n}+\mu u_{t}^{n}+\rho u^{n}-h, \mathscr{A} u_{t t}^{n}\right\rangle \\
& =\left\|\sqrt{1-\sigma} \nabla u_{t t}^{n}\right\|_{L^{2}(\Omega)}^{2}+\left\langle-\nabla \sigma u_{t t}^{n}+\nabla\left(c^{2} \mathscr{A} u^{n}+b \mathscr{A}^{\beta} \partial_{t}^{\alpha} u^{n}+\mu u_{t}^{n}+\rho u^{n}-h\right), \nabla u_{t t}^{n}\right\rangle_{L^{2}(\Omega)} \\
& \geq\left\|\sqrt{1-\sigma} \nabla u_{t t}^{n}\right\|_{L^{2}(\Omega)}^{2}-\frac{1}{2}(1-\bar{\sigma})\left\|\nabla u_{t t}^{n}\right\|_{L^{2}(\Omega)}^{2} \\
& \quad-\frac{1}{2(1-\bar{\sigma})} \|-\nabla \sigma u_{t t}^{n}+c^{2} \nabla \mathscr{A} u^{n}+b \nabla \mathscr{A}^{\beta} \partial_{t}^{\alpha} u^{n} \\
& \quad+\nabla \mu u_{t}^{n}+\mu \nabla u_{t}^{n}+\nabla \rho u^{n}+\rho \nabla u^{n}-\nabla h \|_{L^{2}(\Omega)}^{2}
\end{aligned}
$$

which yields

$$
\begin{aligned}
& \left\|\nabla u_{t t}^{n}\right\|_{L_{t}^{2}\left(L^{2}\right)} \leq \frac{1}{1-\bar{\sigma}} \\
& \quad\left(\|\nabla \sigma\|_{L^{\infty}\left(L^{4}\right)}\left\|u_{t t}^{n}\right\|_{L^{2}\left(L^{4}\right)}^{2}+c^{2}\left\|\mathscr{A}^{3 / 2} u^{n}\right\|_{L_{t}^{2}\left(L^{2}\right)}+b\left\|\mathscr{A}^{1 / 2+\beta} \partial_{t}^{\alpha} u^{n}\right\|_{L_{t}^{2}\left(L^{2}\right)}\right. \\
& \quad+\|\nabla \mu\|_{L^{2}\left(L^{4}\right)}\left\|u_{t}^{n}\right\|_{L^{\infty}\left(L^{4}\right)}+\|\mu\|_{L_{t}^{2}\left(L^{\infty}\right)}\left\|\nabla u_{t}^{n}\right\|_{L_{t}^{\infty}\left(L^{2}\right)} \\
& \left.\quad+\|\nabla \rho\|_{L_{t}^{2}\left(L^{2}\right)}\left\|u^{n}\right\|_{L_{t}^{\infty}\left(L^{\infty}\right)}+\|\rho\|_{L_{t}^{2}\left(L^{\infty}\right)}\left\|\nabla u^{n}\right\|_{L_{t}^{\infty}\left(L^{2}\right)}\right)
\end{aligned}
$$

where we can again employ the embedding estimates (23) and assume

$$
\|\nabla \sigma\|_{L^{\infty}\left(L^{4}\right)}<\frac{1-\bar{\sigma}}{C_{H^{1} \rightarrow L^{4}}}
$$

in order to extract an estimate of the form

$$
\begin{aligned}
& \left\|\nabla u_{t t}^{n}\right\|_{L_{t}^{2}\left(L^{2}\right)} \\
& \leq C\left(\left\|\mathscr{A}^{3 / 2} u^{n}\right\|_{L_{t}^{2}\left(L^{2}\right)}+\left\|\mathscr{A}^{1 / 2+\beta} \partial_{t}^{\alpha} u^{n}\right\|_{L_{t}^{2}\left(L^{2}\right)}+\left\|\nabla u_{t}^{n}\right\|_{L_{t}^{\infty}\left(L^{2}\right)}+\left\|\nabla u^{n}\right\|_{L_{t}^{\infty}\left(L^{2}\right)}\right)
\end{aligned}
$$

Adding a multiple (factor $\left.\varepsilon\left(\|\triangle \sigma\|_{L^{\infty}\left(L^{4}\right)}^{2}\left(C_{H^{1}, L^{4}}\right)^{2}+\|\nabla \sigma\|_{L_{t}^{\infty}\left(L^{\infty}\right)}^{2}\right)\right)$ of the square of (25) to (22), making $\varepsilon$ small enough (so that $C^{2} \varepsilon<\frac{b}{2 \Gamma(\alpha) t^{1-\alpha}}$ ) and all terms containing $L^{\infty}(0, T)$ norms of $u^{n}$ on the right hand side of (22) can be dominated by left hand side terms) using the fact that $1 / 2+\beta \leq 1+\beta / 2$ for $\beta \in[0,1]$ and Gronwall's inequality we end up with an estimate of the form

$$
\begin{aligned}
& \left\|\nabla u_{t t}^{n}\right\|_{L^{2}\left(L^{2}\right)}^{2}+\left\|\mathscr{A} u_{t}^{n}\right\|_{L_{t}^{\infty}\left(\dot{L}^{2}\right)}^{2}+\left\|\mathscr{A}^{1+\beta / 2} \partial_{t}^{\alpha} u^{n}\right\|_{L_{t}^{2}\left(\dot{L}^{2}\right)}^{2}+\left\|\mathscr{A}^{3 / 2} u^{n}\right\|_{L_{t}^{\infty}\left(\dot{L}^{2}\right)}^{2} \\
& \leq C(T)\left(\left\|\mathscr{A} u_{t}^{n}(0)\right\|_{\dot{L}^{2}(\Omega)}^{2}+\left\|\mathscr{A}^{3 / 2} u^{n}(0)\right\|_{\dot{L}^{2}(\Omega)}^{2}+\|\mathscr{A} h\|_{L_{t}^{2}\left(\dot{L}^{2}\right)}^{2}\right)
\end{aligned}
$$

which via weak limits shows existence of a solution to the homogeneous initial boundary value problem for (16) and transfers to $u$ as

$$
\begin{aligned}
\|u\|_{U}^{2} & :=\left\|\nabla u_{t t}\right\|_{L^{2}\left(L^{2}\right)}^{2}+\left\|\mathscr{A} u_{t}\right\|_{L_{t}^{\infty}\left(\dot{L}^{2}\right)}^{2}+\left\|\mathscr{A}^{1+\beta / 2} \partial_{t}^{\alpha} u\right\|_{L_{t}^{2}\left(\dot{L}^{2}\right)}^{2}+\left\|\mathscr{A}^{3 / 2} u\right\|_{L_{t}^{\infty}\left(\dot{L}^{2}\right)}^{2} \\
& \leq C(T)\left(\left\|\mathscr{A} u_{t}(0)\right\|_{\dot{L}^{2}(\Omega)}^{2}+\left\|\mathscr{A}^{3 / 2} u(0)\right\|_{\dot{L}^{2}(\Omega)}^{2}+\|\mathscr{A} h\|_{L_{t}^{2}\left(\dot{L}^{2}\right)}^{2}\right)
\end{aligned}
$$

The required regularity on $\sigma, \mu, \rho, h, u_{0}, u_{1}$ is, besides (18) and (24)

$$
\begin{aligned}
& \sigma \in H^{1}\left(0, T ; L^{\infty}(\Omega)\right) \cap L^{\infty}\left(0, T ; W^{2,4}(\Omega) \cap W^{1, \infty}(\Omega)\right) \\
& \mu \in L^{2}\left(0, T ; H^{2}(\Omega)\right), \quad \rho \in L^{2}\left(0, T ; H^{2}(\Omega)\right) \\
& h \in L^{2}\left(0, T ; \dot{H}^{2}(\Omega)\right), \quad u_{0} \in \dot{H}^{3}(\Omega), \quad u_{1} \in \dot{H}^{2}(\Omega)
\end{aligned}
$$


Proposition 3.1. Under conditions (18), (24), (28), there exists a unique solution

$$
u \in U:=H^{2}\left(0, T ; \dot{L}^{2}(\Omega)\right) \cap W^{1, \infty}\left(0, T ; \dot{H}^{2}(\Omega)\right) \cap L^{\infty}\left(0, T ; \dot{H}^{3}(\Omega)\right)
$$

to the initial boundary value problem (16), (17). This solution satisfies the estimate (27).

The energy estimate leading to this result has been obtained by basically "multiplying (16) with $\mathscr{A}^{2} u_{t}$ ", that is, taking the $\dot{L}^{2}(\Omega)$ inner product of the PDE with $\mathscr{A}^{2} u_{t}$ and using selfadjointness of $\mathscr{A}$ in $\dot{L}^{2}(\Omega)$.

Later on, we will also need less regular solutions along with estimates on them. Since the proofs are actually somewhat simpler then, we skip the details on Galerkin approximation and only provide the energy estimates.

Multiplying (16) with $\mathscr{A} u_{t}$ we obtain

$$
\begin{aligned}
& \frac{1}{2}\left\|\sqrt{1-\sigma(t)} \nabla u_{t}(t)\right\|_{L^{2}(\Omega)}^{2}+\frac{c^{2}}{2}\|\mathscr{A} u(t)\|_{\dot{L}^{2}(\Omega)}^{2}+\frac{1}{2 \Gamma(\alpha) t^{1-\alpha}}\left\|\mathscr{A}^{(1+\beta) / 2} \partial_{t}^{\alpha} u\right\|_{L_{t}^{2}\left(\dot{L}^{2}\right)}^{2} \\
& \leq \frac{1}{2}\left\|\sqrt{1-\sigma(0)} \nabla u_{t}(0)\right\|_{L^{2}(\Omega)}^{2}+\frac{c^{2}}{2}\|\mathscr{A} u(0)\|_{\dot{L}^{2}(\Omega)}^{2} \\
& -\int_{0}^{t}\left\langle\frac{1}{2} \sigma_{t}(s) \nabla u_{t}(s)+\nabla \sigma(s) u_{t t}(s)+\nabla \mu u_{t}+\mu \nabla u_{t}+\nabla \rho u+\rho \nabla u-\nabla h, \nabla u_{t}(s)\right\rangle_{L^{2}(\Omega)} d s \\
& \leq \frac{1}{2}\left\|\sqrt{1-\sigma(0)} \nabla u_{t}(0)\right\|_{L^{2}(\Omega)}^{2}+\frac{c^{2}}{2}\|\mathscr{A} u(0)\|_{\dot{L}^{2}(\Omega)}^{2}+\frac{1}{2 \varepsilon}\left\|\nabla u_{t}\right\|_{L_{t}^{2}\left(L^{2}\right)}^{2} \\
& +\frac{\varepsilon}{2}\left(\frac{1}{2}\left\|\sigma_{t}\right\|_{L_{t}^{2}\left(L^{\infty}\right)}\left\|\nabla u_{t}\right\|_{L_{t}^{\infty}\left(L^{2}\right)}+\|\nabla \sigma\|_{L_{t}^{\infty}\left(L^{\infty}\right)}\left\|u_{t t}\right\|_{L_{t}^{2}\left(L^{2}\right)}\right. \\
& \quad+\|\nabla \mu\|_{L^{2}\left(L^{4}\right)}\left\|u_{t}\right\|_{L^{\infty}\left(L^{4}\right)}+\|\mu\|_{L_{t}^{2}\left(L^{\infty}\right)}\left\|\nabla u_{t}\right\|_{L_{t}^{\infty}\left(L^{2}\right)} \\
& \left.\quad+\|\nabla \rho\|_{L_{t}^{2}\left(L^{2}\right)}\|u\|_{L_{t}^{\infty}\left(L^{\infty}\right)}+\|\rho\|_{L^{2}\left(L^{4}\right)}\|\nabla u\|_{L^{\infty}\left(L^{4}\right)}+\|\nabla h\|_{L_{t}^{2}\left(L^{2}\right)}\right)^{2} .
\end{aligned}
$$

The PDE provides us with

$$
\begin{aligned}
& \left\|u_{t t}\right\|_{L_{t}^{2}\left(L^{2}\right)}=\left\|-\frac{1}{1-\sigma}\left(c^{2} \mathscr{A} u+b \mathscr{A}^{\beta} \partial_{t}^{\alpha} u+\mu u_{t}+\rho u-h\right)\right\|_{L^{2}\left(L^{2}\right)} \\
& \leq C\left(\|\mu\|_{L_{t}^{2}\left(L^{\infty}\right)}+\|\rho\|_{L^{2}\left(L^{4}\right)}\right) \times \\
& \quad \sup _{t \in(0, T)}\left(\left\|\sqrt{1-\sigma(t)} \nabla u_{t}(t)\right\|_{L^{2}(\Omega)}^{2}+\frac{c^{2}}{2}\|\mathscr{A} u(t)\|_{\dot{L}^{2}(\Omega)}^{2}+\frac{1}{2 \Gamma(\alpha) t^{1-\alpha}}\left\|\mathscr{A}^{\beta} \partial_{t}^{\alpha} u\right\|_{L_{t}^{2}\left(\dot{L}^{2}\right)}^{2}\right)
\end{aligned}
$$

which, due to the condition $\beta \leq(1+\beta) / 2$, allows us to dominate the $\varepsilon u_{t t}$ term on the right hand side of (30). Thus, using Gronwall's inequality, we get an estimate of the form

$$
\begin{aligned}
& \|u\|_{U_{l o}}^{2}:=\left\|u_{t t}\right\|_{L_{t}^{2}\left(\dot{L}^{2}\right)}^{2}+\left\|\nabla u_{t}\right\|_{L_{t}^{\infty}\left(\dot{L}^{2}\right)}^{2}+\left\|\mathscr{A}^{(1+\beta) / 2} \partial_{t}^{\alpha} u\right\|_{L_{t}^{2}\left(\dot{L}^{2}\right)}^{2}+\|\mathscr{A} u\|_{L_{t}^{\infty}\left(\dot{L}^{2}\right)}^{2} \\
& \leq C(T)\left(\left\|\nabla u_{t}(0)\right\|_{L^{2}(\Omega)}^{2}+\|\mathscr{A} u(0)\|_{\dot{L}^{2}(\Omega)}^{2}+\|\nabla h\|_{L^{2}\left(L^{2}\right)}^{2}\right)
\end{aligned}
$$

provided

$$
\begin{aligned}
& \sigma \in H^{1}\left(0, T ; L^{\infty}(\Omega)\right) \cap L^{\infty}\left(0, T ; W^{1, \infty}(\Omega)\right) \\
& \mu \in L^{2}\left(0, T ; L^{\infty}(\Omega) \cap W^{1,4}(\Omega)\right), \quad \rho \in L^{2}\left(0, T ; H^{1}(\Omega)\right) \\
& h \in L^{2}\left(0, T ; H^{1}(\Omega)\right), \quad u_{0} \in \dot{H}^{2}(\Omega), \quad u_{1} \in \dot{H}^{1}(\Omega)
\end{aligned}
$$

Proposition 3.2. Under the conditions (18), (32), there exists a unique solution $u$, where $u \in H^{2}\left(0, T ; \dot{L}^{2}(\Omega)\right) \cap W^{1, \infty}\left(0, T ; \dot{H}^{1}(\Omega)\right) \cap L^{\infty}\left(0, T ; \dot{H}^{2}(\Omega)\right)$, to the initial boundary value 
problem (16), (17), and this solution satisfies the estimate (31).

An even lower regularity estimate can be obtained by multiplication of (16) with $u_{t}$, which yields

$$
\begin{aligned}
& \frac{1}{2}\left\|\sqrt{1-\sigma(t)} u_{t}(t)\right\|_{\dot{L}^{2}(\Omega)}^{2}+\frac{c^{2}}{2}\|\nabla u(t)\|_{L^{2}(\Omega)}^{2}+\frac{1}{2 \Gamma(\alpha) t^{1-\alpha}}\left\|\mathscr{A}^{\beta / 2} \partial_{t}^{\alpha} u\right\|_{L_{t}^{2}\left(\dot{L}^{2}\right)}^{2} \\
& \leq \frac{1}{2}\left\|\sqrt{1-\sigma(0)} u_{t}(0)\right\|_{\dot{L}^{2}(\Omega)}^{2}+\frac{c^{2}}{2}\|\nabla u(0)\|_{L^{2}(\Omega)}^{2} \\
& \quad-\int_{0}^{t}\left\langle\frac{1}{2} \sigma_{t}(s) u_{t}(s)+\mu u_{t}+\rho u-h, u_{t}(s)\right\rangle d s \\
& \leq \frac{1}{2}\left\|\sqrt{1-\sigma(0)} u_{t}(0)\right\|_{\dot{L}^{2}(\Omega)}^{2}+\frac{c^{2}}{2}\|\nabla u(0)\|_{L^{2}(\Omega)}^{2}+\frac{1}{2 \varepsilon}\left\|u_{t}\right\|_{L_{t}^{2}\left(\dot{L}^{2}\right)}^{2} \\
& \quad+\frac{\varepsilon}{2}\left(\frac{1}{2}\left\|\sigma_{t}\right\|_{L_{t}^{2}\left(L^{\infty}\right)}\left\|u_{t}\right\|_{L_{t}^{\infty}\left(\dot{L}^{2}\right)}+\|\mu\|_{L_{t}^{2}\left(L^{\infty}\right)}\left\|u_{t}\right\|_{L_{t}^{\infty}\left(\dot{L}^{2}\right)}\right. \\
& \left.\quad+\|\rho\|_{L^{2}\left(L^{4}\right)}\|u\|_{L^{\infty}\left(L^{4}\right)}+\|h\|_{L_{t}^{2}\left(\dot{L}^{2}\right)}\right)^{2},
\end{aligned}
$$

(where we have used the fact that $\left\|\frac{c}{c_{0}}\right\|_{L^{\infty}(\Omega)} \leq 1$ ), hence an estimate of the form

$$
\begin{aligned}
& \|u\|_{U_{v l}}^{2}:=\left\|u_{t}\right\|_{L_{t}^{\infty}\left(\dot{L}^{2}\right)}^{2}+\left\|\mathscr{A}^{\beta / 2} \partial_{t}^{\alpha} u\right\|_{L^{2}\left(L^{2}\right)}^{2}+\|\nabla u\|_{L_{t}^{\infty}\left(L^{2}\right)}^{2} \\
& \leq C(T)\left(\left\|u_{t}(0)\right\|_{\dot{L}^{2}(\Omega)}^{2}+\|\nabla u(0)\|_{L^{2}(\Omega)}^{2}+\|h\|_{L_{t}^{2}\left(\dot{L}^{2}\right)}^{2}\right)
\end{aligned}
$$

Here it obviously suffices to assume

$$
\begin{aligned}
& \sigma \in H^{1}\left(0, T ; L^{\infty}(\Omega)\right), \quad \mu \in L_{t}^{2}\left(L^{\infty}\right), \quad \rho \in L^{2}\left(L^{4}\right) \\
& h \in L_{t}^{2}\left(\dot{L}^{2}\right), \quad u_{0} \in \dot{H}^{1}(\Omega), \quad u_{1} \in \dot{L}^{2}(\Omega)
\end{aligned}
$$

Proposition 3.3. Under conditions (18) and (34), there exists a unique solution $u$ lying in the space $u \in H^{2}\left(0, T ; \dot{H}^{-1}(\Omega)\right) \cap W^{1, \infty}\left(0, T ; \dot{L}^{2}(\Omega)\right) \cap L^{\infty}\left(0, T ; \dot{H}^{1}(\Omega)\right)$ and satisfying the initial boundary value problem (16), (17). This solution satisfies the estimate (33).

We proceed to proving well-posedness of the nonlinear problem (3) with $D=b \mathscr{A}^{\beta} \partial_{t}^{\alpha}$ by applying a fixed point argument to the operator $\mathscr{T}$ mapping $v$ to the solution of

$$
\begin{aligned}
(1-2 \kappa v) u_{t t}+c^{2} \mathscr{A} u+b \mathscr{A}^{\beta} \partial_{t}^{\alpha} u-2 \kappa v_{t} u_{t} & =r \text { in } \Omega \times(0, T) \\
u(0)=0, \quad u_{t}(0) & =0 \text { in } \Omega
\end{aligned}
$$

that is, of (16), (17) with $\sigma(x, t)=-2 \kappa(x) v(x, t), \mu(x, t)=-2 \kappa(x) v_{t}(x, t), \rho(x, t)=0$, $h(x, t)=r(x, t)$. We first consider self-mapping of $\mathscr{T}$. Even in case of constant $\kappa$, the regularity requirements on $\sigma, \mu, \rho$ force us into the high regularity scenario of Proposition 3.1. For spatially variable $\kappa$, due to the estimates

$$
\begin{aligned}
& \|\Delta \mu\|_{L^{2}\left(L^{2}\right)}=2\left\|\triangle\left(\kappa v_{t}\right)\right\|_{L^{2}\left(L^{2}\right)} \\
& \quad \leq 2\left(\|\triangle \kappa\|_{L^{2}(\Omega)}\left\|v_{t}\right\|_{L^{2}\left(0, T ; L^{\infty}(\Omega)\right.}+2\|\nabla \kappa\|_{L^{4}(\Omega)}\left\|\nabla v_{t}\right\|_{L^{2}\left(L^{4}\right)}+\|\kappa\|_{L^{\infty}(\Omega)}\left\|\Delta v_{t}\right\|_{L^{2}\left(L^{2}\right)}\right) \\
& \left\|\sigma_{t}\right\|_{L_{t}^{2}\left(L^{\infty}\right)} \leq 2 C_{H^{2}, L^{\infty}}\left\|\triangle\left(\kappa v_{t}\right)\right\|_{L^{2}\left(L^{2}\right)} \\
& \|\triangle \sigma\|_{L^{\infty}\left(L^{4}\right)}=2\|\triangle(\kappa v)\|_{L^{\infty}\left(L^{4}\right)} \\
& \quad \leq 2\left(\|\triangle \kappa\|_{L^{4}(\Omega)}\|v\|_{L^{\infty}\left(L^{\infty}\right)}+2\|\nabla \kappa\|_{L^{4}(\Omega)}\|\nabla v\|_{L^{\infty}\left(L^{\infty}\right)}+\|\kappa\|_{L^{\infty}(\Omega)}\|\Delta v\|_{L^{\infty}\left(L^{4}\right)}\right) \\
& \|\nabla \sigma\|_{L_{t}^{\infty}\left(L^{\infty}\right)}=2\|\nabla(\kappa v)\|_{L_{t}^{\infty}\left(L^{\infty}\right)} \leq 2\left(\|\nabla \kappa\|_{L^{\infty}(\Omega)}\|v\|_{L^{\infty}\left(L^{\infty}\right)}+\|\kappa\|_{L^{\infty}(\Omega)}\|\nabla v\|_{L^{\infty}\left(L^{\infty}\right)}\right)
\end{aligned}
$$


the regularity

$$
\kappa \in W^{2,4}(\Omega) \cap W^{1, \infty}(\Omega)
$$

is sufficient for obtaining the regularity (28) for any for any $v \in U$. To achieve the nondegeneracy and smallness conditions (18), (24), we use the estimates

$$
\begin{aligned}
& \|\sigma\|_{L_{t}^{\infty}\left(L^{\infty}\right)} \leq 2\|\kappa\|_{L^{\infty}(\Omega)}\|v\|_{L_{t}^{\infty}\left(L^{\infty}\right)} \\
& \|\nabla \sigma\|_{L^{\infty}\left(L^{4}\right)} \leq 2\|\nabla \kappa\|_{L^{4}(\Omega)}\|v\|_{L^{\infty}\left(L^{\infty}\right)}+2\|\kappa\|_{L^{\infty}(\Omega)}\|\nabla v\|_{L^{\infty}\left(L^{4}\right)}
\end{aligned}
$$

and additionally to (36) require smallness of $v$.

Proposition 3.1 yields that $\mathscr{T}$ is a self-mapping on

$$
B_{R}=\left\{v \in U:\|v\|_{U} \leq R\right\}
$$

provided the initial and right hand side data are sufficiently small so that

$$
C(T)\left(\left\|\mathscr{A} u_{1}\right\|_{\dot{L}^{2}(\Omega)}^{2}+\left\|\mathscr{A}^{3 / 2} u_{0}\right\|_{\dot{L}^{2}(\Omega)}^{2}+\|\mathscr{A} r\|_{L_{t}^{2}\left(\dot{L}^{2}\right)}^{2}\right) \leq R^{2}
$$

with $C(T)$ as in Proposition 3.1. In view of (18), (24), (37), we choose $R$ such that

$$
\left(C_{H^{1} \rightarrow L^{4}}\left(\|\nabla \kappa\|_{L^{4}(\Omega)} C_{H^{2}, L^{\infty}}+\|\kappa\|_{L^{\infty}(\Omega)} C_{H^{1}, L^{4}}\right)+\|\kappa\|_{L^{\infty}(\Omega)} C_{H^{2}, L^{\infty}}\right) R<\frac{1}{2} .
$$

Contractivity of $\mathscr{T}$ can be shown by taking $v^{(1)}$ and $v^{(2)}$ in $B_{R}$ and considering $u^{(1)}=$ $\mathscr{T} v^{(1)}$ and $u^{(2)}=\mathscr{T} v^{(2)}$, whose differences $\bar{u}=u^{(1)}-u^{(2)}$ and $\bar{v}=v^{(1)}-v^{(2)}$ solve

$$
\left(1-2 \kappa v^{(1)}\right) \bar{u}_{t t}+c^{2} \mathscr{A} \bar{u}+b \mathscr{A}^{\beta} \partial_{t}^{\alpha} \bar{u}_{t}-2 \kappa v_{t}^{(1)} \bar{u}_{t}=2 \kappa \bar{v}_{t} u_{t}^{(2)}+2 \kappa \bar{v} u_{t t}^{(2)}
$$

with homogeneous initial conditions. Similarly to above, with $\sigma=-2 \kappa v^{(1)}, \mu=-2 \kappa v_{t}^{(1)}$, $\rho(x, t)=0, h=2 \kappa \bar{v}_{t} u_{t}^{(2)}+2 \kappa \bar{v} u_{t t}^{(2)}$, since $v^{(1)}, u^{(2)} \in B_{R}$ (the latter due to the already shown self-mapping property of $\mathscr{T}$ ) we satisfy the conditions (18), (24), (28) on $\sigma$ and $\mu$. However $h$ in general fails to be contained in $L^{2}\left(0, T ; \dot{H}^{2}(\Omega)\right.$ ) (in particular the term $\left.2 \kappa \bar{v} u_{t t}^{(2)}\right)$, hence we move to the lower order regularity regime from Proposition 3.2 . To this end, we estimate

$$
\|\nabla h\|_{L^{2}\left(L^{2}\right)} \leq 2\|\kappa\|_{L^{4}(\Omega)}\left(\left\|\bar{v}_{t}\right\|_{L^{2}\left(L^{4}\right)}\left\|u_{t}^{(2)}\right\|_{L_{t}^{\infty}\left(L^{\infty}\right)}+\|\bar{v}\|_{L_{t}^{\infty}\left(L^{\infty}\right)}\left\|u_{t t}^{(2)}\right\|_{L^{2}\left(L^{4}\right)}\right) .
$$

Thus imposing the additional smallness condition

$$
\theta:=2\|\kappa\|_{L^{4}(\Omega)} \sqrt{C} C_{H^{1}, L^{4}} C_{H^{2}, L^{\infty}}(\sqrt{T}+1) R<1
$$

on $R$ and employing from Proposition 3.2 , we obtain contractivity

$$
\left\|\mathscr{T} v^{(1)}-\mathscr{T} v^{(2)}\right\|_{U_{l o}}=\|\bar{u}\|_{U_{l o}} \leq \theta\|\bar{v}\|_{U_{l o}}=\theta\left\|v^{(1)}-v^{(2)}\right\|_{U_{l o}}
$$

Theorem 3.1. For any $\alpha \in(0,1), T>0, \kappa \in W^{2,4}(\Omega) \cap W^{1, \infty}(\Omega)$ there exists $R_{0}>0$ such that for any data $u_{0} \in \dot{H}^{3}(\Omega), u_{1} \in \dot{H}^{2}(\Omega), r \in L^{2}\left(0, T ; \dot{H}^{2}(\Omega)\right)$ satisfying

$$
\left\|\mathscr{A} u_{1}\right\|_{\dot{L}^{2}(\Omega)}^{2}+\left\|\mathscr{A}^{3 / 2} u_{0}\right\|_{\dot{L}^{2}(\Omega)}^{2}+\|\mathscr{A} r\|_{L^{2}\left(\dot{L}^{2}\right)}^{2} \leq R_{0}^{2}
$$

there exists a unique solution $u \in U$ of

$$
\begin{aligned}
(1-2 \kappa u) u_{t t}+c^{2} \mathscr{A} u+b \mathscr{A}^{\beta} \partial_{t}^{\alpha} u & =2 \kappa\left(u_{t}\right)^{2}+r \text { in } \Omega \times(0, T) \\
u(0)=u_{0}, \quad u_{t}(0) & =u_{1} \text { in } \Omega .
\end{aligned}
$$

Existence of the linearisation of $G$ requires well-posedness of (9) with $D=b \mathscr{A} \partial_{t}^{\alpha}$, that is, (16), (17) with $\sigma=-2 \kappa u, \mu=-4 \kappa u_{t}, \rho=-2 \kappa u_{t t}, h=2 \underline{\delta \kappa}\left(u u_{t t}+u_{t}^{2}\right)$. Due to the appearance of a $u_{t t}$ term we are in a similar situation to the contractivity proof above 
and therefore the lower regularity Proposition 3.2 is the right framework for analysing the linearisation of the forward problem.

Proposition 3.4. Under the assumptions of Theorem 3.1 for any $\underline{\delta} \kappa \in W^{1, \infty}(\Omega)$ there exists a unique solution $z \in U_{l o}$ of

$$
\begin{aligned}
(1-2 \kappa u) z_{t t}+c^{2} \mathscr{A} z+b \mathscr{A}^{\beta} \partial_{t}^{\alpha} z-4 \kappa u_{t} z_{t}-2 \kappa u_{t t} z & =2 \underline{\delta \kappa}\left(u u_{t t}+u_{t}^{2}\right) \text { in } \Omega \times(0, T) \\
z(0)=0, \quad z_{t}(0) & =0 \text { in } \Omega,
\end{aligned}
$$

where $u \in U$ solves (42).

In order to prove Fréchet differentiability we also need to bound the solution of (11) that is, (16), (17) with $\sigma=-2 \kappa u, \mu=-4 \kappa u_{t}, \rho=-2 \kappa u_{t t}, h=2 \underline{d \kappa}\left(v \tilde{u}_{t t}+u v_{t t}+\left(\tilde{u}_{t}+u_{t}\right) v_{t}\right)+$ $2 \kappa\left(v v_{t t}+v_{t}^{2}\right)$, where $v$ can be bounded analogously to $z$ by Proposition 3.2, in particular we can only expect to have $v_{t t} \in L^{2}\left(0, T ; L^{2}(\Omega)\right)$, so $h \in L^{2}\left(0, T ; H^{1}(\Omega)\right)$ is out of reach and we show Fréchet differentiability in the very low regularity regime of Proposition 3.3 .

Theorem 3.2. For any $\alpha \in(0,1), T>0, \bar{R}>0$ there exists $R_{0}>0$ such that for any data $u_{0} \in \dot{H}^{3}(\Omega), u_{1} \in \dot{H}^{2}(\Omega), \quad r \in L^{2}\left(0, T ; \dot{H}^{2}(\Omega)\right)$ satisfying (41), the parameter-tostate map $G: B_{\bar{R}}(0) \rightarrow U$ is well-defined according to Theorem 3.1 Moreover, it is Fréchet differentiable as an operator $G: B_{\bar{R}}(0) \rightarrow U_{v l}$. Here $B_{\bar{R}}(0)=\left\{\kappa \in W^{2,4}(\Omega) \cap W^{1, \infty}(\Omega)\right.$ : $\left.\|\kappa\|_{W^{2,4}(\Omega) \cap W^{1, \infty}(\Omega)} \leq \bar{R}\right\}$.

\subsection{Fractional Zener damping}

Consider

$$
D=b_{1} \mathscr{A} \partial_{t}^{\alpha_{1}}+b_{2} \partial_{t}^{\alpha_{2}+2} \quad \text { with } b_{2}>0, b_{1} \geq b_{2} c^{2}, 1 \geq \alpha_{1} \geq \alpha_{2}>0,
$$

where based on the analysis in [17] we expect to get well-posedness of the nonlinear forward problem only in case $\alpha_{1}=1$, so we first of all focus on this case. Later on we will also prove a well-posedness result on the equation linearized at $\kappa=0$ in the practically relevant case $\alpha_{1}=\alpha_{2}=: \alpha$.

Again we first of all consider the initial boundary value problem for the general linear PDE

$$
\begin{aligned}
& (1-\sigma) u_{t t}+c^{2} \mathscr{A} u+b_{1} \mathscr{A} u_{t}+b_{2} \partial_{t}^{\alpha_{2}+2} u+\mu u_{t}+\rho u=h \\
& u(0)=u_{0}, \quad u_{t}(0)=u_{1}, \quad\left(u_{t t}(0)=u_{2} \text { in case } \alpha_{2}>\frac{1}{2}\right)
\end{aligned}
$$

with given space- and time dependent functions $\sigma, \mu, \rho, h$.

Again we skip the details about the Faedo-Galerkin approach and the discretisation index $n$ and only provide the crucial energy estimate. We multiply (44) with $\mathscr{A} u_{t t}$ and integrate with respect to time, using the inequalities and identities

$$
\int_{0}^{t}\left\langle\mathscr{A} u(s), \mathscr{A} u_{t t}(s)\right\rangle d s=\left\langle\mathscr{A} u(t), \mathscr{A} u_{t}(t)\right\rangle-\left\langle\mathscr{A} u(0), \mathscr{A} u_{t}(0)\right\rangle-\int_{0}^{t}\left\|\mathscr{A} u_{t}(s)\right\|_{\dot{L}^{2}(\Omega)}^{2}
$$

and

$$
\begin{aligned}
& \int_{0}^{t}\left\langle\partial_{t}^{\alpha_{2}+2}[u](s), \mathscr{A} u_{t t}(s)\right\rangle d s=\int_{0}^{t}\left\langle\partial_{t}^{\alpha_{2}}\left[\nabla u_{t t}\right](s), \nabla u_{t t}(s)\right\rangle d s \\
& \geq \frac{1}{2} \int_{0}^{t} \partial_{t}^{\alpha_{2}}\left[\left\|\nabla u_{t t}\right\|_{\dot{L}^{2}(\Omega)}^{2}\right](s) d s=\frac{1}{2} I_{t}^{1-\alpha}\left[\left\|\nabla u_{t t}\right\|_{L^{2}(\Omega)}^{2}\right](t) \geq \frac{1}{2 \Gamma(1-\alpha) t^{\alpha}}\left\|\nabla u_{t t}\right\|_{L_{t}^{2}\left(L^{2}\right)}^{2},
\end{aligned}
$$

where the latter equality holds provided $u_{t t}(0)=0$ and we have applied (14) (actually to the Fourier components of the Galerkin discretisation $\left.w=\lambda_{j}^{1 / 2} u_{j}^{n \prime \prime}\right)$ with $\gamma=\alpha_{2}$. 
This yields the energy estimate

$$
\begin{aligned}
& \left(\frac{b_{2}}{2 \Gamma(1-\alpha) t^{\alpha}}+1-\bar{\sigma}\right)\left\|\nabla u_{t t}\right\|_{L_{t}^{2}\left(L^{2}\right)}^{2}+\frac{b_{1}}{2}\left\|\mathscr{A} u_{t}(t)\right\|_{\dot{L}^{2}(\Omega)}^{2} \\
& \leq \frac{b_{1}}{2}\left\|\mathscr{A} u_{t}(0)\right\|_{L_{t}^{2}\left(\dot{L}^{2}\right)}^{2}+c^{2}\left\langle\mathscr{A} u(0), \mathscr{A} u_{t}(0)\right\rangle \\
& \quad-c^{2}\left\langle\mathscr{A} u(t), \mathscr{A} u_{t}(t)\right\rangle+c^{2}\left\|\mathscr{A} u_{t}\right\|_{L_{t}^{2}\left(\dot{L}^{2}\right)}^{2}+\int_{0}^{t}\left\langle\nabla \sigma u_{t t}+\nabla\left(\mu u_{t}+\rho u-h\right)(s), \nabla u_{t t}(s)\right\rangle d s \\
& \leq \frac{b_{1}}{2}\left\|\mathscr{A} u_{t}(0)\right\|_{L_{t}^{2}\left(\dot{L}^{2}\right)}^{2}+c^{2}\left\langle\mathscr{A} u(0), \mathscr{A} u_{t}(0)\right\rangle \\
& \quad+\frac{b_{1}}{4}\left\|\mathscr{A} u_{t}(t)\right\|_{\dot{L}^{2}(\Omega)}^{2}+\frac{c^{4}}{b_{1}}\|\mathscr{A} u(t)\|_{\dot{L}^{2}(\Omega)}^{2}+c^{2}\left\|\mathscr{A} u_{t}\right\|_{L_{t}^{2}\left(\dot{L}^{2}\right)}^{2} \\
& \quad+\|\nabla \sigma\|_{L^{\infty}\left(L^{4}\right)}\left\|u_{t t}\right\|_{L^{2}\left(L^{4}\right)}\left\|\nabla u_{t t}\right\|_{L_{t}^{2}\left(L^{2}\right)}+\frac{\varepsilon}{2}\left\|\nabla u_{t t}\right\|_{L_{t}^{2}\left(L^{2}\right)}^{2} \\
& \quad+\frac{1}{2 \varepsilon}\left(\|\nabla \mu\|_{L_{t}^{\infty}\left(L^{2}\right)}\left\|u_{t}\right\|_{L^{2}\left(0, t ; L^{\infty}(\Omega)\right.}+\|\mu\|_{L^{\infty}\left(L^{4}\right)}\left\|\nabla u_{t}\right\|_{L^{2}\left(L^{4}\right)}\right. \\
& \left.\quad+\|\nabla \rho\|_{L_{t}^{2}\left(L^{2}\right)}\|u\|_{L_{t}^{\infty}\left(L^{\infty}\right)}+\|\rho\|_{L^{2}\left(L^{4}\right)}\|\nabla u\|_{L^{\infty}\left(L^{4}\right)}+\|\nabla h\|_{L_{t}^{2}\left(L^{2}\right)}\right)^{2}
\end{aligned}
$$

Here we assume nondegeneracy

$$
\sigma(x, t) \leq \bar{\sigma}<\frac{b_{2}}{\Gamma(1-\alpha) T^{\alpha}}+1 \text { for all } x \in \Omega \quad t \in(0, T) .
$$

and smallness of $\nabla \sigma$

$$
\|\nabla \sigma\|_{L^{\infty}\left(L^{4}\right)}<\frac{\frac{b_{2}}{\Gamma(1-\alpha) T^{\alpha}}+1-\bar{\sigma}}{C_{H^{1} \rightarrow L^{4}}}
$$

and choose $\varepsilon<\frac{b_{2}}{\Gamma(1-\alpha) T^{\alpha}}+1-\underline{\sigma}-C_{H^{1} \rightarrow L^{4}}\|\nabla \sigma\|_{L^{\infty}\left(L^{4}\right)}$ to obtain, using Gronwall's Lemma,

$$
\left\|\nabla u_{t t}\right\|_{L^{2}\left(L^{2}\right)}^{2}+\left\|\mathscr{A} u_{t}\right\|_{L_{t}^{\infty}\left(\dot{L}^{2}\right)}^{2} \leq C\left(\left\|\mathscr{A} u_{t}(0)\right\|_{\dot{L}^{2}(\Omega)}^{2}+\|\nabla h\|_{L_{t}^{2}\left(\dot{L}^{2}\right)}^{2}\right)
$$

The required regularity on $\sigma, \mu, \rho, h, u_{0}, u_{1}$, is, besides (46), (47)

$$
\begin{aligned}
& \mu \in L^{\infty}\left(0, T ; H^{1}(\Omega)\right), \quad \rho \in L^{2}\left(0, T ; H^{1}(\Omega)\right), \\
& h \in L^{2}\left(0, T ; \dot{H}^{1}(\Omega)\right), \quad u_{0}, u_{1} \in \dot{H}^{2}(\Omega), \quad u_{2}=0 .
\end{aligned}
$$

Proposition 3.5. Under conditions (46), (47), (49), there exists a unique solution

$$
u \in U:=H^{2}\left(0, T ; \dot{H}^{1}(\Omega)\right) \cap W^{1, \infty}\left(0, T ; \dot{H}^{2}(\Omega)\right)
$$

to the initial boundary value problem (44), (45), and this solution satisfies the estimate (48).

Theorem 3.3. For any $\alpha_{2} \in(0,1), T>0, \kappa \in W^{1,4}(\Omega)$ there exists $R_{0}>0$ such that for any data $u_{0}, u_{1} \in \dot{H}^{2}(\Omega), r \in L^{2}\left(0, T ; \dot{H}^{1}(\Omega)\right)$ satisfying

$$
\left\|\mathscr{A} u_{1}\right\|_{\dot{L}^{2}(\Omega)}^{2}+\left\|\mathscr{A} u_{0}\right\|_{\dot{L}^{2}(\Omega)}^{2}+\|\nabla r\|_{L^{2}\left(L^{2}\right)}^{2} \leq R_{0}^{2}
$$

there exists a unique solution $u \in U$ of

$$
\begin{gathered}
b_{2} \partial_{t}^{\alpha_{2}+2} u+(1-2 \kappa u) u_{t t}+c^{2} \mathscr{A} u+b_{1} \mathscr{A} u_{t}=2 \kappa\left(u_{t}\right)^{2}+r \text { in } \Omega \times(0, T) \\
u(0)=u_{0}, \quad u_{t}(0)=u_{1}, \quad u_{t t}=0 \text { in } \Omega .
\end{gathered}
$$

Proposition 3.6. Under the assumptions of Theorem 3.3 for any $\underline{\delta \kappa} \in L^{3}(\Omega)$ there exists 
a unique solution $z \in U$ of

$$
\begin{gathered}
b_{2} \partial_{t}^{\alpha_{2}+2} z+(1-2 \kappa u) z_{t t}+c^{2} \mathscr{A} z+b_{1} \mathscr{A} z_{t}-4 \kappa u_{t} z_{t}-2 \kappa u_{t t} z=2 \underline{\delta \kappa}\left(u u_{t t}+u_{t}^{2}\right) \text { in } \Omega \times(0, T) \\
z(0)=0, \quad z_{t}(0), \quad z_{t t}=0=0 \text { in } \Omega .
\end{gathered}
$$

where $u \in U$ solves (52).

Theorem 3.4. For any $\alpha_{2} \in(0,1), T>0, \bar{R}>0$ there exists $R_{0}>0$ such that for any data $u_{0}, u_{1} \in \dot{H}^{2}(\Omega), r \in L^{2}\left(0, T ; \dot{H}^{2}(\Omega)\right)$ satisfying (51), the parameter-to-state map $G$ : $B_{\bar{R}}(0) \rightarrow U$ is well-defined according to Theorem 3.3 Moreover, it is Fréchet differentiable as an operator $G: B_{\bar{R}}(0) \rightarrow U$. Here $B_{\bar{R}}(0)=\left\{\kappa \in W^{1,4}(\Omega):\|\kappa\|_{W^{1,4}(\Omega)} \leq \bar{R}\right\}$.

We now consider the linear problem in case $\alpha_{1}=\alpha_{2}=: \alpha, b_{1}=b_{2} c^{2}+\delta$ with $\delta \geq 0$

$$
(1-\sigma) u_{t t}+c^{2} \mathscr{A} u+b_{1} \mathscr{A} \partial_{t}^{\alpha} u+b_{2} \partial_{t}^{\alpha+2} u+\mu u_{t}+\rho u=h
$$

in which the differential operator can partially be factorised as

$$
\begin{aligned}
& (1-\sigma) \partial_{t t}+c^{2} \mathscr{A}+\left(b_{2} c^{2}+\delta\right) \mathscr{A} \partial_{t}^{\alpha}+b_{2} \partial_{t}^{\alpha+2}+\mu \partial_{t}+\rho \mathrm{id} \\
& =\left(\partial_{t t}+c^{2} \mathscr{A}\right)\left(b_{2} \partial_{t}^{\alpha}+\mathrm{id}\right)-\sigma \partial_{t t}+\delta \mathscr{A} \partial_{t}^{\alpha}+\mu \partial_{t}+\rho \mathrm{id}
\end{aligned}
$$

Thus, up to the "perturbation" terms containing $\sigma, \delta, \mu$, and $\rho$, the auxiliary function $\tilde{u}=$ $b_{2} \partial_{t}^{\alpha} u+u$ satisfies a wave equation $\tilde{u}_{t t}+c^{2} \mathscr{A} \tilde{u}=h$. Motivated by this fact, we multiply (54) with $\mathscr{A} \tilde{u}_{t}$ to obtain the energy identity

$$
\begin{aligned}
\frac{1}{2}\left\|\nabla\left(b_{2} \partial_{t}^{\alpha} u+u\right)_{t}(t)\right\|_{L^{2}(\Omega)}^{2}+ & \frac{c^{2}}{2}\left\|\mathscr{A}\left(b_{2} \partial_{t}^{\alpha} u+u\right)(t)\right\|_{\dot{L}^{2}(\Omega)}^{2} \\
& +\delta \int_{0}^{t}\left\langle\nabla \partial_{t}^{\alpha} u(s), \nabla\left(b_{2} \partial_{t}^{\alpha} u+u\right)_{t}(s)\right\rangle d s \\
=\frac{1}{2}\left\|\nabla\left(b_{2} \partial_{t}^{\alpha} u+u\right)_{t}(0)\right\|_{L^{2}(\Omega)}^{2} & +\frac{c^{2}}{2}\left\|\mathscr{A}\left(b_{2} \partial_{t}^{\alpha} u+u\right)(0)\right\|_{\dot{L}^{2}(\Omega)}^{2} \\
& +\int_{0}^{t}\left\langle\nabla\left(h+\sigma u_{t} t-\mu u_{t}-\rho u\right)(s), \nabla\left(b_{2} \partial_{t}^{\alpha} u+u\right)_{t}(s)\right\rangle d s .
\end{aligned}
$$

The term containing $\delta$ can be nicely tackled by means of Lemma 3.1

$$
\begin{aligned}
\int_{0}^{t}\left\langle\nabla \partial_{t}^{\alpha} u(s)\right. & \left., \nabla\left(b_{2} \partial_{t}^{\alpha} u+u\right)_{t}(s)\right\rangle d s \\
& =\frac{b_{2}}{2}\left\|\nabla \partial_{t}^{\alpha} u(t)\right\|_{L^{2}(\Omega)}^{2}-\frac{b_{2}}{2}\left\|\nabla \partial_{t}^{\alpha} u(0)\right\|_{L^{2}(\Omega)}^{2}+\int_{0}^{t}\left\langle\nabla \partial_{t}^{\alpha} u(s), \nabla u_{t}(s)\right\rangle d s \\
& \geq \frac{b_{2}}{2}\left\|\nabla \partial_{t}^{\alpha} u(t)\right\|_{L^{2}(\Omega)}^{2}+\frac{1}{2 \Gamma(\alpha) t^{1-\alpha}}\left\|\nabla \partial_{t}^{\alpha} u\right\|_{L_{t}^{2}\left(L^{2}\right)}^{2},
\end{aligned}
$$

(which reflects the physical fact that $\delta$ is the diffusivity of sound and therefore the corresponding term models damping). However, in the term containing $\sigma$ this is inhibited by the time-dependence of $\sigma$. Thus in case $\alpha_{1}=\alpha_{2}=: \alpha<1$ we have to restrict ourselves to the linearisation of the forward problem at $\kappa=0$ (where also $\mu=0, \rho=0$ ), where the 
above together with Young's inequality yields the energy estimate

$$
\begin{aligned}
& \frac{1}{2}\left\|\nabla\left(b_{2} \partial_{t}^{\alpha} u+u\right)_{t}\right\|_{L_{t}^{\infty}\left(L^{2}\right)}^{2}+c^{2}\left\|\mathscr{A}\left(b_{2} \partial_{t}^{\alpha} u+u\right)\right\|_{L_{t}^{\infty}\left(\dot{L}^{2}\right)}^{2} \\
& \quad+\delta b_{2}\left\|\nabla \partial_{t}^{\alpha} u(t)\right\|_{L_{t}^{\infty}\left(L^{2}\right)}^{2}+\frac{1}{2 \Gamma(\alpha) t^{1-\alpha}}\left\|\nabla \partial_{t}^{\alpha} u\right\|_{L_{t}^{2}\left(L^{2}\right)}^{2} \\
& \quad \leq\left\|\nabla\left(b_{2} \partial_{t}^{\alpha} u+u\right)_{t}(0)\right\|_{L^{2}(\Omega)}^{2}+c^{2}\left\|\mathscr{A}\left(b_{2} \partial_{t}^{\alpha} u+u\right)(0)\right\|_{\dot{L}^{2}(\Omega)}^{2}+\frac{1}{2}\|\nabla h\|_{L_{t}^{1}\left(L^{2}\right)}^{2}
\end{aligned}
$$

The PDE yields an estimate of $\tilde{u}_{t t}$ as follows

$$
\left\|\left(b_{2} \partial_{t}^{\alpha} u+u\right)_{t t}\right\|_{L_{t}^{\infty}\left(L^{2}\right)}=\left\|h-c^{2} \mathscr{A}\left(b_{2} \partial_{t}^{\alpha} u+u\right)\right\|_{L_{t}^{\infty}\left(\dot{L}^{2}\right)}^{2}
$$

To extract temporal reguarity of $u$ from regularity of $b_{2} \partial_{t}^{\alpha} u+u$ for $b_{2}>0$, we make use of known regularity results of time fractional ODEs: $b_{2} \partial_{t}^{\alpha} u+u=\tilde{u} \in W^{k, \infty}(0, T ; Z)$ implies $u \in W^{k+\alpha, \infty}(0, T ; Z)$.

In order to formulate a result on this linearisation, we will postulate the regularity of $u$ that is to be "expected" from (56), (57) (but apparently not provable):

$$
u \in U:=W^{2+\alpha, \infty}\left(0, T ; \dot{L}^{2}(\Omega)\right) \cap W^{1+\alpha, \infty}\left(0, T ; \dot{H}^{1}(\Omega)\right) \cap W^{\alpha, \infty}\left(0, T ; \dot{H}^{2}(\Omega)\right)
$$

The required regularity of $\underline{\delta \kappa}$ to guarantee $h=2 \underline{\delta \kappa}\left(u u_{t t}+u_{t}^{2}\right) \in L^{1}\left(0, t ; L^{2}(\Omega)\right)$ is therefore $\underline{\delta \kappa} \in L^{\infty}(\Omega)$.

We thus obtain the following result on the linearisation of the forward problem at $\kappa=0$ :

Proposition 3.7. For any $u \in U$ and $\underline{\delta \kappa} \in L^{\infty}(\Omega)$ there exists a unique solution $z \in U$ of

$$
\begin{gathered}
b_{2} \partial_{t}^{\alpha+2} z+z_{t t}+c^{2} \mathscr{A} z+b_{1} \mathscr{A} \partial_{t}^{\alpha} z=2 \underline{\delta \kappa}\left(u u_{t t}+u_{t}^{2}\right) \text { in } \Omega \times(0, T) \\
z(0)=0, \quad z_{t}(0), \quad z_{t t}=0=0 \text { in } \Omega .
\end{gathered}
$$

\section{Injectivity of the linearised forward operator}

The forwards map is defined by $F(\kappa)=\operatorname{tr}_{\Sigma} u$, where $\operatorname{tr}_{\Sigma} v$ denotes the time trace of the space and time dependent function $v:(0, T) \times \Omega$ at the observation surface $\Sigma$ (which may also just be a single point $\left.\Sigma=\left\{x_{0}\right\}\right)$ and $u$ solves

$$
\begin{aligned}
& u_{t t}+c^{2} \mathscr{A} u+D u=\kappa(x)\left(u^{2}\right)_{t t}+r \text { in } \Omega \times(0, T) \\
& u(0)=0, \quad u_{t}(0)=0 \text { in } \Omega .
\end{aligned}
$$

Its linearisation at $\kappa=0$ in direction $\underline{\delta \kappa}$ is $F^{\prime}(0) \underline{\delta \kappa}=\operatorname{tr}_{\Sigma} z_{0}$, where $z_{0}$ solves

$$
z_{t t}+c^{2} \mathscr{A} z+D z=\underline{\delta \kappa}\left(u_{0}^{2}\right)_{t t}
$$

where

$$
u_{0, t}+c^{2} \mathscr{A} u_{0}+D u_{0}=r .
$$

Both PDEs (60), (61) come with homogeneous initial conditions.

As in the previous section, we consider the two damping models

$$
D=b \mathscr{A}^{\beta} \partial_{t}^{\alpha} \quad(\mathrm{CWCH})
$$

and

$$
D=b_{1} \mathscr{A} \partial_{t}^{\alpha_{1}}+b_{2} \partial_{t}^{\alpha_{2}+2} \quad(\mathrm{FZ})
$$


The Laplace transformed solutions to the corresponding resolvent equation

$$
\hat{w}(\lambda, s)=\frac{1}{\omega(\lambda, s)} \text { with } \omega(\lambda, s)=\left\{\begin{array}{l}
s^{2}+b \lambda^{\beta} s^{\alpha}+c^{2} \lambda \text { for } \mathrm{CWCH} \\
b_{2} s^{2+\alpha_{2}}+s^{2}+b_{1} \lambda s^{\alpha_{1}}+c^{2} \lambda \text { for FZ }
\end{array}\right.
$$

will play a crucial role in the proofs below.

From [17, Lemma 4.1] we conclude that in case of $\mathrm{CWCH}$, the function $\omega^{c w c h}(\lambda, \cdot)$ has precisely two complex-conjugate zeros $p_{+}^{c w c h}(\lambda), p_{-}^{c w c h}(\lambda)$, which lie in the left hand complex plane.

For FZ, we first consider the particular parameter configuration (corresponding to vanishing viscosity of sound)

$$
b_{1}=b_{2} c^{2} \text { and } \alpha_{1}=\alpha_{2}
$$

in which we can factorise $\omega^{F Z}(\lambda, s)=\left(b_{2} s^{\alpha_{2}}+1\right)\left(s^{2}+c^{2} \lambda\right)$ and get the roots

$$
p_{0}^{F Z}=-\frac{1}{b_{2}}\left(\text { only in case } \alpha_{1}=\alpha_{2}=1\right), \quad p_{ \pm}^{F Z}(\lambda)= \pm i c \sqrt{\lambda}
$$

Note that $p_{0}^{F Z}$ is independent of $\lambda$, but $p_{ \pm}^{F Z}(\lambda)$ obviously allows to distinguish between different $\lambda$ 's. This distinction is possible in general, a fact that has already been shown for the $\mathrm{CWCH}$ case with $\beta=1$ in [17, Remark 4.1]. As an additional result, that is not needed for the uniqueness proof but might be convenient for the computation of poles and residues, we state that the poles are single in certain cases.

Lemma 4.1. The poles of $\hat{w}^{\text {cwch }}$ and of $\hat{w}^{F Z}$ (except for $p_{0}^{F Z}$ in case $\alpha_{1}=\alpha_{2}=1$ ) differ for different $\lambda$. Moreover, in the case $\mathrm{CWCH}$ and in the case $\mathrm{FZ}$ with (64) the poles are single.

Proof. For CWCH, let $f(z)=z^{2}+c^{2} \lambda, g(z)=b \lambda^{\beta} z^{\alpha}$. Then for a sufficiently large $R>c^{2} \lambda$ let $C_{R}$ be the circle radius $R$, centre at the origin. Then $|g(z)|<|f(z)|$ on $C_{R}$ and so Rouché's theorem shows that $f(z)$ and $(f+g)(z)$ have the same number of roots, counted with multiplicity, within $C_{R}$. For $f$ these are only at $z= \pm i \sqrt{\lambda} c$ so the same must be true of $f+g$ and so $\omega^{c w c h}$ has precisely one single root in the third and in the fourth quadrant, respectively.

Suppose now that $\hat{w}^{c w c h}$ has a pole at $r e^{i \theta}$, where $\pi / 2<\theta<\pi$, for both $\lambda_{1}$ and $\lambda_{2}$. Then for $s=r e^{i \theta}$

$$
s^{2}+b \lambda_{1}^{\beta} s^{\alpha}+c^{2} \lambda_{1}=0 \quad s^{2}+b \lambda_{2}^{\beta} s^{\alpha}+c^{2} \lambda_{2}=0
$$

so that

$$
\frac{b}{c^{2}} \frac{\left(\lambda_{1}^{\beta}-\lambda_{2}^{\beta}\right)}{\left(\lambda_{1}-\lambda_{2}\right)}=-s^{\alpha}
$$

Now if $\lambda_{1} \neq \lambda_{2}$ then the left hand side is positive and real and so $\alpha \theta=\pi$. This means that $\theta>\pi$, a contradiction.

In case of FZ, assuming that $p$ is a pole of both $\hat{w}^{F Z}\left(\lambda_{1}, \cdot\right)$ and $\hat{w}^{F Z}\left(\lambda_{2}, \cdot\right)$ we have

$$
0=\omega\left(\lambda_{1}, p\right)-\omega\left(\lambda_{2}, p\right)=\left(\lambda_{1}-\lambda_{2}\right)\left[b_{1} p^{\alpha_{1}}+c^{2}\right],
$$

where due $\omega\left(\lambda_{1}, p\right)=0$, the term in brackets $b_{1} p^{\alpha_{1}}+c^{2}=-\frac{p^{2}}{\lambda_{1}}\left(b_{2} p^{\alpha_{2}}+1\right) \neq 0$, hence $\lambda_{1}=\lambda_{2}$. In the factorisable case (64) of FZ, obviously all roots are single. 
As in [16] (where we used the classical damping term $D=b \mathscr{A} \partial_{t}$ ), we assume that $r$ has the form

$$
r(x, t)=f(x) \chi^{\prime \prime}(t)+c^{2} \mathscr{A} f(x) \chi(t)+D[f(x) \chi(t)]
$$

with some function $f$ in the domain of $\mathscr{A}$ vanishing only on a set of measure zero and some twice differentiable function $\chi$ of time such that $\left(\chi^{2}\right)^{\prime \prime}\left(t_{0}\right) \neq 0$ for some $t_{0}>0$. With (65), the solution $u_{0}$ of equation (61) is clearly given by $u_{0}(x, t)=f(x) \chi(t)$, so that $\underline{d \kappa}\left(u_{0}^{2}\right)_{t t}$ can be written in the form

$$
\left(\underline{d \kappa}(x) u_{0}^{2}(x, t)\right)_{t t}=\sum_{j=1}^{\infty} a_{j} \phi_{j}(x) \psi_{j}(t)
$$

where $a_{j}$ are the coefficients of $\underline{d \kappa} \cdot f$ with respect to the eigenfunction basis $\left(\phi_{j}\right)_{j \in \mathbb{N}}$, and $\psi_{j}=\left(\chi^{2}\right)^{\prime \prime}$.

We can rewrite equation (60) as

$$
z_{j}^{\prime \prime}(t)+c^{2} \lambda_{j} z_{j}(t)+D_{j} z_{j}=a_{j} \psi_{j}(t), \quad t>0, \quad z_{j}(0)=0, z_{j}^{\prime}(0)=0
$$

for all $j \in \mathbb{N}$, where

$$
z_{0}(x, t)=\sum_{j=1}^{\infty} z_{j}(t) \phi_{j}(x), \quad D_{j}=\left\{\begin{array}{l}
b \lambda_{j}^{\beta} \partial_{t}^{\alpha} \text { for } \mathrm{CWCH} \\
b_{2} \partial_{t}^{2+\alpha_{2}}+b_{1} \lambda_{j} \partial_{t}^{\alpha_{1}} \text { for FZ }
\end{array}\right.
$$

Applying the Laplace transform to both sides of (67) yields

$$
\hat{z}_{j}(s)=\hat{w}_{j}(s) a_{j} \widehat{\psi}_{j}(s), \quad \text { where } \hat{w}_{j}(s)=\frac{1}{\omega\left(\lambda_{j}, s\right)}, \quad s \in \mathbb{C},
$$

and we have used homogeneity of the initial conditions.

Thus, assuming that $F^{\prime}(0) \underline{d \kappa}=\operatorname{tr}_{\Sigma} z_{0}=0$ implies that

$$
0=\hat{z}_{0}\left(x_{0}, s\right)=\sum_{j=1}^{\infty} a_{j} \phi_{j}\left(x_{0}\right) \hat{w}_{j}(s) \widehat{\psi}_{j}(s), \quad \text { for all } s \in \mathbb{C}, x_{0} \in \Sigma .
$$

Considering the residues at some pole $p_{m}$ corresponding to the eigenvalue $\lambda_{m}$ and using the fact that by Lemma4.1, $\left(s-p_{m}\right) \hat{w}\left(\lambda_{j}, s\right)=0$ for $j \neq m$ yields

$$
\begin{aligned}
0 & =\operatorname{Res}\left(\hat{z}_{0}\left(x_{0} ; p_{m}\right)\right)=\sum_{j=1}^{\infty} a_{j} \phi_{j}\left(x_{0}\right) \lim _{s \rightarrow p_{m}}\left(s-p_{m}\right)^{\ell_{m}} \hat{w}\left(\lambda_{j}, s\right) \widehat{\psi}_{j}(s) \\
& =\operatorname{Res}\left(\hat{w}_{m} ; p_{m}\right) \widehat{\psi}_{j}\left(p_{m}\right) \sum_{k \in K_{m}} a_{k} \phi_{k}\left(x_{0}\right) .
\end{aligned}
$$

Here $\ell_{m}$ is the multiplicity of $p_{m}$ as a root of $\omega\left(\lambda_{m}, \cdot\right)$ and $K_{m} \subseteq \mathbb{N}$ is an enumeration of the eigenspace basis $\left(\phi_{k}\right)_{k \in K_{m}}$ corresponding to the eigenvalue $\lambda_{m}$. Assuming now that

$$
\widehat{\psi}_{k}\left(p_{m}\right) \neq 0
$$

and there exists points $x_{0, m, 1}, \ldots x_{0, m, N_{m}} \in \Sigma, N_{m} \geq \# K_{m}$ such that

$$
\text { the matrix } \phi_{k}\left(x_{0, m, i}\right)_{k \in K_{m}, i \in\left\{1, \ldots, N_{m}\right\}} \text { has full rank \# } K_{m}
$$

we can conclude that $a_{k}=0$ for all $k \in K_{m}$.

Now since $\left(u_{0}^{2}\right)_{t t}\left(t_{0}\right)=f\left(\chi^{2}\right)^{\prime \prime}\left(t_{0}\right)$ only vanishes on a set of measure zero and (66), we can conclude that $\underline{d \kappa}=0$ almost everywhere.

Theorem 4.1. Under the above assumptions (66), (69), (70) for all $m \in \mathbb{N}, k \in K_{m}$, the linearised derivative at $\kappa=0, F^{\prime}(0)$ is injective. 
In particular, (70) is satisfied in the spatially 1-dimensional case $\Sigma=\left\{x_{0}\right\}$, where all eigenvalues of $\mathscr{A}$ are single, i.e., $\# K_{m}=1$ for all $m$, provided none of the eigenfunctions vanish at $x_{0}$; this can be achieved by taking $x_{0}$ on the boundary and where $\phi_{j}$ is subject to nonDirichlet conditions.

\section{Ill-posedness of the linearised inverse problem}

As in the injectivity section, we consider the linear(ised at $\kappa=0$ ) problem of recovering $\underline{\delta \kappa}(x)$ from time trace observations

$$
h(t)=\operatorname{tr}_{\Sigma} z_{0}=z_{0}\left(x_{0}, t_{0}\right), \quad x_{0} \in \Sigma,
$$

where $z_{0}$ solves

$$
z_{t t}+c^{2} \mathscr{A} z+D z=\underline{\delta \kappa}\left(u_{0}^{2}\right)_{t t}
$$

with $u_{0}$ solving

$$
u_{0, t t}+c^{2} \mathscr{A} u_{0}+D u_{0}=r
$$

both with homogeneous initial conditions.

Again, we assume that the excitation $r$ has been chosen such that $u_{0}$ takes the form $u_{0}(x, t)=f(x) \chi(t)$ and employ the shorthand notation $\tilde{\chi}=\left(\chi^{2}\right)^{\prime \prime}$. Using the eigensystem of $\mathscr{A}$ we can then write

$$
z_{0}(x, t)=\sum_{j=1}^{\infty} \phi(x) z_{j}(t)
$$

where

$$
\hat{z}_{j}(s)=\hat{w}_{j}(s)\left\langle\underline{\delta \kappa} \cdot f, \phi_{j}\right) \widehat{\tilde{\chi}}(s), \quad \hat{w}_{j}(s)=\frac{1}{\omega\left(\lambda_{j}, s\right)}, \quad s \in \mathbb{C},
$$

and

$$
\omega(\lambda, s)=\left\{\begin{array}{l}
s^{2}+b \lambda^{\beta} s^{\alpha}+c^{2} \lambda \text { for } \mathrm{CWCH} \\
b_{2} s^{2+\alpha_{2}}+s^{2}+b_{1} \lambda s^{\alpha_{1}}+c^{2} \lambda \text { for FZ }
\end{array}\right.
$$

As in the injectivity section we obtain (for simplicity in the 1-d case where all eigenvalues are single)

$$
\operatorname{Res}\left(\hat{h}\left(p_{m}\right)\right)=\operatorname{Res}\left(\hat{w}_{m} ; p_{m}\right) \widehat{\tilde{\chi}}\left(p_{m}\right)\left\langle\underline{\delta \kappa} \cdot f, \phi_{m}\right\rangle \phi_{m}\left(x_{0}\right)
$$

that is,

$$
\left\langle\underline{\boldsymbol{\delta} \kappa} \cdot f, \phi_{m}\right\rangle=\operatorname{Res}\left(\hat{h}\left(p_{m}\right)\right)\left(\operatorname{Res}\left(\hat{w}_{m} ; p_{m}\right) \widehat{\tilde{\chi}}\left(p_{m}\right) \phi_{m}\left(x_{0}\right)\right)^{-1}
$$

(In our numerical example we had $\chi(t)=t$, hence $\widehat{\tilde{\chi}}\left(p_{m}\right)=2$.)

By l'Hospital's rule we have

$\operatorname{Res}\left(\hat{w}_{m} ; p_{m}\right)=\lim _{s \rightarrow p_{m}} \frac{s-p_{m}}{\omega(\lambda, s)}=\lim _{s \rightarrow p_{m}} \frac{1}{\omega^{\prime}(\lambda, s)}=\left\{\begin{array}{l}\frac{1}{2 p_{m}+\alpha b \lambda_{j}^{\beta} p_{m}^{\alpha-1}} \text { for } \mathrm{CWCH} \\ \frac{1}{\left(2+\alpha_{2}\right) b_{2} p_{m}^{1+\alpha_{2}}+2 p_{m}+\alpha_{1} b_{1} \lambda_{j} p_{m}^{\alpha_{1}-1}} \text { for FZ. }\end{array}\right.$

Thus, the factor multiplied with $\left\langle\underline{\delta \kappa} \cdot f, \phi_{m}\right\rangle$ in (74) only mildly grows with $p_{m}$.

The major ill-posedness seems to lie in the evaluation of the residue of the observations $\operatorname{Res}\left(\hat{h}\left(p_{m}\right)\right)$ at the poles $p_{m}$, form knowledge of $h(t)$ for $t>0$, that is, from $\hat{h}(s)=$ $\int_{0}^{\infty} e^{-s t} h(t) d t$ for $s$ with nonnegative real part (so that the integral defining the Laplace 
transform is well-defined). If these poles lie on the imaginary axis (wave equation), this is still well posed. The further left the poles lie, the more ill-posed this problem.

\subsection{Location of the poles of the relaxation functions}

Motivated by their role for the degree of ill-posedness of the inverse problem, we develop some further results - beyond those stated in Lemma 4.1 as essentials for our uniqueness proof - for each of the two models under consideration and also provide some computational results with plots of these poles for several parameter configurations.

The $\mathrm{CWCH}$ model: Poles of the $\mathrm{CWCH}$ model are the roots of the function

$$
\omega_{\mathrm{CWCH}}(s):=s^{2}+b \lambda^{\beta} s^{\alpha}+c^{2} \lambda=0 .
$$

We recall that according to [17, Lemma 4.1] which relies on Rouché's theorem, they lie in the left hand complex plane and this is easily shown by the following alternative argument.

Suppose $s$ is a root in the first quadrant. Then $\mathbf{s}$, the line joining the origin to the point $s$ can be split into a component in the direction of the positive real axis and one in the direction of the positive imaginary axis. Then since $\alpha \leq 1, \mathbf{s}^{\alpha}$ has components in the same directions. Similarly, the vector $\mathbf{s}^{2}$ has a component parallel to the real axis and again one in the direction of the positive imaginary axis. Since $\lambda \geq 0$, the same is true of the vector $b \lambda^{\beta} \mathbf{s}^{\alpha}$. The third vector repre-

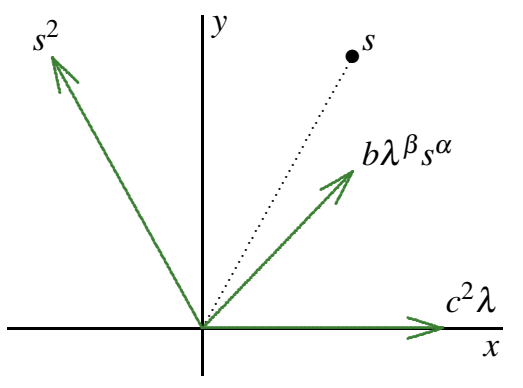
senting $c^{2} \lambda \mathbf{x}$ points along the real axis. However, the sum of these three vectors cannot add to zero contradicting the claim the root $s$ lay in the first quadrant. An identical argument shows $s$ cannot lie in the fourth quadrant and hence cannot lie in the right half plane.

For $b=0$ the poles are along the imaginary axis and spaced exactly as the eigenvalue sequence $\left\{\lambda_{n}\right\}$ stretched by the factor $c^{2}$. As $b$ increases so does the (negative) real component of the poles which follow a curve whose rough slope is determined by the ratio of $b$ and $c^{2}$. The powers $\alpha$ and $\beta$ also are a factor that influence the skewness of the curve along which the poles align. The magnitude of the real and imaginary parts show the relative strengths of the damping and oscillation effects respectively in the equation.

The roots of $\omega_{\mathrm{CWCH}}(s)$ are shown in Figure 1 with $b=0.1, \lambda_{n}=n^{2} \pi^{2}$, and for both $c=1$ and $c=5$, as well as $\alpha=\frac{1}{2}$ and $\alpha=\frac{9}{10}$ and illustrate the above point.

Some notes on how these poles were computed. For rational $\alpha=p / q, \omega_{\mathrm{CWCH}}(s)$ can be written as $z^{2 q}+B z^{p}+C$ with $B=b \lambda^{\beta}, C=c^{2} \lambda$ and where $s=z^{p}$. Now the $2 q^{\text {th }}$ degree polynomial can be represented as the characteristic polynomial of a $2 q \times 2 q$ matrix. Then the roots of this polynomial are calculated by computing the eigenvalues of the companion matrix. This gives a good approximation even for reasonably large $q$ values but additional care must be taken, see, for example, [10]. Given now the values of $\left\{z_{n}\right\}$ for $\lambda \in\left\{\lambda_{n}\right\}$, one can recover $\left\{s_{n}\right\}$ from $s_{n}=z_{n}^{p}$. This is subject to considerable round-off error for even modest values of $p$. However it is usually sufficient as an initial approximation for Newton's method to then compute a more exact value of the roots of $\omega$ to desired accuracy. This is also successful for real $\alpha$ by first taking a rational approximation $\alpha \approx p / q$ for the 


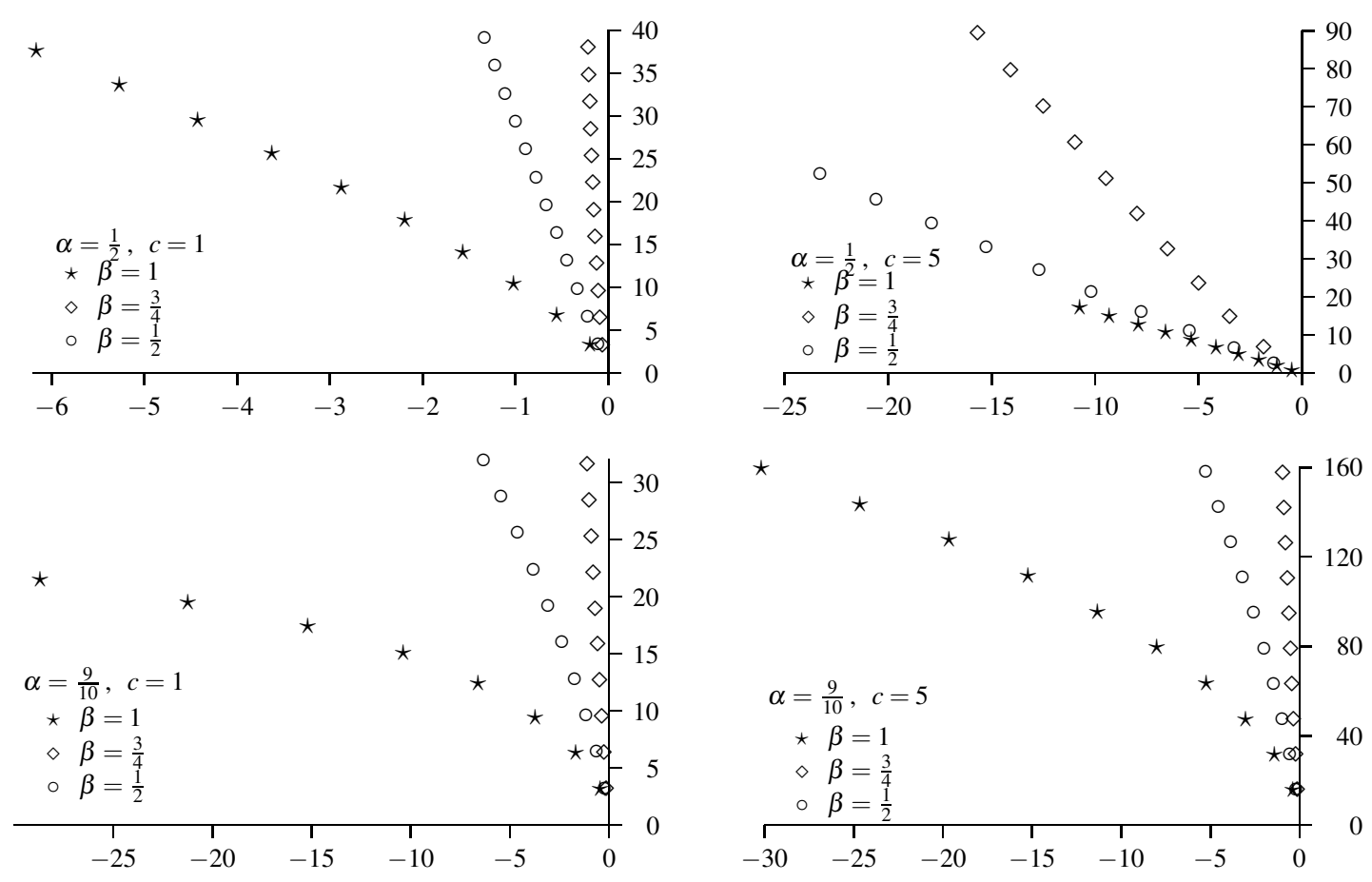

Figure 1: Roots of $\omega_{\mathrm{CWCH}}(s)$ for various $\alpha, \beta, c$ values.

initial approximation of the roots and then proceeding as above.

The FZ model: Poles of the fractional Zener model are the roots of the function

$$
\omega_{\mathrm{FZ}}(s):=b_{2} s^{2+\alpha_{2}}+s^{2}+b_{1} \lambda s^{\alpha_{1}}+c^{2} \lambda=0 .
$$

There is a more complex relationship here and more constants whose value can affect the outcome. In the case that $\alpha_{1}=\alpha_{2}=\alpha$, we can re-write this as

$$
\omega_{\mathrm{FZ}}(s):=\left(b_{2} s^{\alpha}+1\right)\left(s^{2}+c^{2} \lambda\right)+\delta \lambda s^{\alpha}=0, \quad \text { where } \delta:=b_{1}-c^{2} b_{2}
$$

and $\delta$ needs to be nonnegative, cf. [12, Section III.B]. If $\delta=0$ then $\omega_{\mathrm{FZ}}(s)$ factors. There will be two roots at $\pm i \sqrt{\lambda} c$ on the imaginary axis and a potential root coming from $s^{\alpha}+1 / b_{2}=0$. The latter only exists in case $\alpha=1$ for otherwise writing $s=r e^{i \theta}$ with $\theta \in(-\pi, \pi]$ we have that $\alpha \theta \in(-\pi, \pi)$ and therefore $\mathfrak{I}\left(s^{\alpha}+1\right)=r^{\alpha} \sin (\alpha \theta)=0$ implies $\alpha \theta=0$, hence $\Re\left(s^{\alpha}+1 / b_{2}\right)=r^{\alpha} \cos (\alpha \theta)+1 / b_{2}>0$. In case $\alpha=1$ we obviously have a root at $-1 / b_{2}$, whose modulus, notably, does not increase with $\lambda$, as opposed to the two other complex conjugate roots of $\omega_{\mathrm{FZ}}$.

Clearly, physical reasoning leads us to the conclusion that in case of a nonegative diffusivity of sound $\delta \geq 0$, all poles need to have nonpositive real part. However, the complex analysis arguments from [17, Lemma 4.1] via Rouché's Theorem, using as a bounding function the dominant power part $f(z)=b_{2} z^{2+\alpha_{2}}+c^{2} \lambda$, does not seem to directly carry over to the FZ case. This is basically due to the fact that we cannot say anything about the number of roots of the non-polynomial function $f$. Additionally, asymptotics in terms of powers of $s$ will be much less effective here since, for small $b_{2}$ and/or $\alpha_{2}$, the term $s^{2}$ will be de facto dominant even for relatively large magnitudes of $s$.

Therefore we have to take a different path to conclude that also in the FZ case, the poles lie in the left hand complex plane. We do so by means of energy estimates similar to those in section 3.2, which basically corresponds to the mentioned physical argument. As a (partial) 
counterpart to [17, Lemma 4.1] we state the following.

Lemma 5.1. The roots of $\omega_{\mathrm{FZ}}(s)$ with $\alpha_{1}=\alpha_{2}=\alpha$ and $\delta:=b_{1}-c^{2} b_{2} \geq 0$ lie in the left hand complex plane.

Proof. We consider the following initial value problem for the relaxation equation

$$
b_{2} \partial_{t}^{2+\alpha_{2}} w+w^{\prime \prime}+b_{1} \lambda \partial_{t}^{\alpha_{1}} c^{2} \lambda w=0, \quad w(0)=0, w^{\prime}(0)=1, \quad w^{\prime \prime}(0)=0 .
$$

The Laplace transform $\hat{w}$ of its solution satisfies

$$
\left(b_{2} s^{2+\alpha_{2}}+s^{2}+b_{1} \lambda s^{\alpha_{1}} c^{2} \lambda\right) \hat{w}(s)=b_{2} s^{\alpha_{2}}+1
$$

and therefore $\hat{w}(s)=\frac{b_{2} s^{\alpha_{2}}+1}{\omega_{\mathrm{FZ}}(s)}$. Now if $\alpha_{1}=\alpha_{2}=\alpha$ and $\delta:=b_{1}-c^{2} b_{2} \geq 0$, analogously to the proof of Proposition 3.7, we obtain an energy estimate for $\tilde{w}:=b_{2} \partial_{t}^{\alpha} w+w$ by multiplying (75) with $w^{\prime \prime}$ and integrating with respect to time

$$
\begin{aligned}
& \frac{1}{2}\left|\tilde{w}_{t}(t)\right|^{2}+\frac{c^{2} \lambda}{2}|\tilde{w}(t)|^{2}+\delta \lambda\left(\frac{b_{2}}{2}\left|\partial_{t}^{\alpha} w(t)\right|^{2}+\frac{1}{2 \Gamma(\alpha) t^{1-\alpha}} \int_{0}^{t}\left|\partial_{t}^{\alpha} w(\tau)\right|^{2} d \tau\right) \\
& \leq \frac{1}{2}\left|\tilde{w}_{t}(0)\right|^{2}+\frac{c^{2} \lambda}{2}|\tilde{w}(0)|^{2}
\end{aligned}
$$

for all $t \geq 0$. This implies uniform boundedness $|\tilde{w}(t)| \leq \sqrt{\left(c^{2} \lambda_{\min }\right)^{-1}\left|\tilde{w}_{t}(0)\right|^{2}+|\tilde{w}(0)|^{2}}=$ : $C$ by a constant independent of $\lambda$. Taking Laplace transforms

$$
|\hat{\tilde{w}}(s)|=\left|\int_{0}^{\infty} e^{-s t} \tilde{w}(t) d t\right| \leq \int_{0}^{\infty} e^{-\Re(s) t} d t C=\frac{C}{\Re(s)}
$$

for $\mathfrak{R}(s)>0$, we see that $\hat{\tilde{w}}(s)$ cannot have any poles in the right half plane. Due to the identity $\hat{\tilde{w}}(s)=\left(b_{2} s^{\alpha}+1\right) \hat{w}(s)-b_{2} s^{\alpha-1} w(0)=\left(b_{2} s^{\alpha}+1\right) \hat{w}(s)=\frac{\left(b_{2} s^{\alpha}+1\right)^{2}}{\omega_{\mathrm{FZ}}(s)}$ (where the numerator has no zeros in case $\alpha \in(0,1))$, the assertion follows.

The effect of $\delta$ on the poles in the FZ model can also be assessed by means of the implicit function theorem, applied to the function

$$
f(r, \theta ; \delta)=\left(\begin{array}{l}
b_{2} r^{2+\alpha_{2}} \cos \left(\left(2+\alpha_{2}\right) \theta\right)+r^{2} \cos (2 \theta)+b_{1} \lambda r^{\alpha_{1}} \cos \left(\alpha_{1} \theta\right)+c^{2} \lambda \\
b_{2} r^{2+\alpha_{2}} \sin \left(\left(2+\alpha_{2}\right) \theta\right)+r^{2} \sin (2 \theta)+b_{1} \lambda r^{\alpha_{1}} \sin \left(\alpha_{1} \theta\right)
\end{array}\right)
$$

whose zeros are the magnitudes and arguments of the roots $s=r e^{i \theta}$ of $\omega^{\mathrm{FZ}}$. Now

$$
\frac{\partial f}{\partial(r, \theta)}=\left(\begin{array}{cc}
A_{1} & A_{2} \\
B_{1} & B_{2}
\end{array}\right), \quad \frac{\partial f}{\partial \delta}=\left(\begin{array}{c}
C_{1} \\
C_{2}
\end{array}\right)
$$

and using Cramer's rule this yields

$$
\frac{\partial r}{\partial \delta}=\frac{B_{1} C_{2}-B_{2} C_{1}}{A_{1} B_{2}-A_{2} B_{1}}, \quad \frac{\partial \theta}{\partial \delta}=\frac{C_{1} A_{2}-C_{2} A_{1}}{A_{1} B_{2}-A_{2} B_{1}},
$$

where $b_{1}=b_{2} c^{2}+\delta$ and for $(r, \theta)$ satisfying $f(r, \theta ; \delta)=0$. Then

$$
\begin{aligned}
A_{1} & =\left(2+\alpha_{2}\right) b_{2} r^{1+\alpha_{2}} \cos \left(\left(2+\alpha_{2}\right) \theta\right)+2 r \cos (2 \theta)+\alpha_{1} b_{1} \lambda r^{\alpha_{1}-1} \cos \left(\alpha_{1} \theta\right) \\
& =-\frac{1}{r}\left(\alpha_{2} r^{2} \cos (2 \theta)+\left(2+\alpha_{2}-\alpha_{1}\right) b_{1} \lambda r^{\alpha_{1}} \cos \left(\alpha_{1} \theta\right)+\left(2+\alpha_{2}\right) c^{2} \lambda\right) \\
A_{2} & =\left(2+\alpha_{2}\right) b_{2} r^{1+\alpha_{2}} \sin \left(\left(2+\alpha_{2}\right) \theta\right)+2 r \sin (2 \theta)+\alpha_{1} b_{1} \lambda r^{\alpha_{1}-1} \sin \left(\alpha_{1} \theta\right) \\
& =-\frac{1}{r}\left(\alpha_{2} r^{2} \sin (2 \theta)+\left(2+\alpha_{2}-\alpha_{1}\right) b_{1} \lambda r^{\alpha_{1}} \sin \left(\alpha_{1} \theta\right)\right) \\
B_{1} & =-r A, \quad B_{2}=r A_{1}, \quad C_{1}=\lambda r^{\alpha_{1}} \cos \left(\alpha_{1} \theta\right), \quad C_{2}=\lambda r^{\alpha_{1}} \sin \left(\alpha_{1} \theta\right) .
\end{aligned}
$$


This results in

$$
\begin{aligned}
& A_{1} B_{2}-A_{2} B_{1}=r\left(A_{1}^{2}+A_{2}^{2}\right) \\
& B_{1} C_{2}-B_{2} C_{1}=\lambda r^{\alpha_{1}}\left(\alpha_{2} r^{2} \cos \left(\left(2-\alpha_{1}\right) \theta\right)+\left(2+\alpha_{2}-\alpha_{1}\right) b_{1} \lambda r^{\alpha_{1}}+\left(2+\alpha_{2}\right) c^{2} \lambda \cos \left(\alpha_{1} \theta\right)\right) \\
& C_{1} A_{2}-C_{2} A_{1}=\lambda r^{\alpha_{1}-1}\left(-\alpha_{2} r^{2} \sin \left(\left(2-\alpha_{1}\right) \theta\right)+\left(2+\alpha_{2}\right) c^{2} \lambda \sin \left(\alpha_{1} \theta\right)\right) .
\end{aligned}
$$

and therefore $\frac{\partial r}{\partial \delta}>0, \quad \frac{\partial \theta}{\partial \delta}>0$, for the case of the known roots $r=c \sqrt{\lambda}, \theta= \pm \pi / 2$ at $\delta=0$, for $\alpha_{1}=\alpha_{2}$. That is, increasing $\delta$ tends to move the poles into the left hand complex plane, which is intuitive in view of its physical role as a diffusivity of sound.

Also here we have employed the method for numerically computing roots as described above. In particular we use this in order to illustrate the influence of $\delta>0$ on the behaviour of the roots, see Figure 2 .
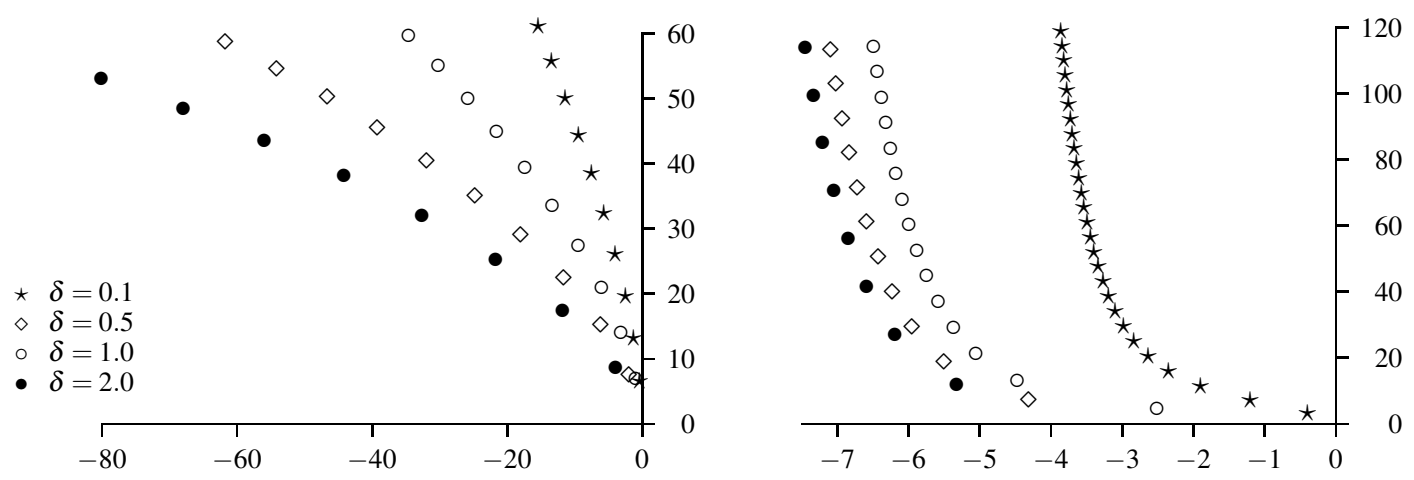

Figure 2: Roots of $\omega_{\mathrm{FZ}}(\mathrm{s})$ for various $\delta$ values with: left $\alpha=\frac{1}{2}$, right $\alpha=\frac{9}{10}$

The location of the poles can thus be computed from knowledge of the constants $b_{1}, b_{2}, c$, the exponents $\alpha$ and $\beta$ as well as the values of $\lambda_{n}$. These poles are also obtainable from the time trace measurements as the zeros of the relaxation function which is the Laplace transform of this data $g$. Thus assuming the spectrum $\left\{\lambda_{n}\right\}_{n=1}^{\infty}$ of $\mathscr{A}$ was known it is perfectly reasonable that a least-squares fit could be made to determine the damping constants contained in the term $D$ appearing in equation (3) and/or the wave speed $c$. While an illconditioned problem, it would be particularly feasible if the time trace data were measured at several points along an arc rather than at a single point. It is further conceivable that spectral information on the eigenvalues of $\mathscr{A}$ could be determined, in particular those of the low frequency. This in turn might be used to obtain knowledge on either a coefficient in $\mathscr{A}$ or on the domain $\Omega$ itself as there is geometrical information contained in the lowest few eigenvalues. See, for example, [11].

\section{Reconstructions of $\kappa$}

The boundary conditions for our test cases were homogeneous Dirichlet at $x=0$ and homogeneous Neumann at $x=1$ with a nonhomogeneous driving term $r(x, t)$ with greater weight near $x=1$. Thus the solution was small in the region near $x=0$ in comparison to near $x=1$ where the data $h(t)=u(1, t)$ was measured. The consequence of this was that $\kappa(x)$ for $x$ small was multiplied by terms that were also small in comparison to that at the rightmost endpoint and resulting in greater ill-conditioning of the inversion near $x=0$. This will be apparent in each of the reconstructions to be shown below. 
The data $h(t)$ was computed by the direct solver at the endpoint $x=1$ and a sample of 50 points were taken to which uniformly distributed random noise was added as representing the actual data measurements that formed the overposed data. This was then filtered by a smoothing routine based on penalising the $H^{2}$ norm with regularisation parameter based on the estimate of the noise and then up-resolved to the working size for the inverse solver.

Discretisation of $\kappa$ was done by means of a fixed set of 40 chapeau basis functions and we applied a regularised frozen Newton iteration, stopped by the discrepancy principle, for numerically solving the discretised inverse problem.

Figure 3 shows the reconstruction of a piecewise linear $\kappa$ for the values $\alpha=1, \alpha=$ $0.9, \alpha=0.5$ and $\alpha=0.25$. In each case the damping coefficient $b$ was kept at $b=$ 0.1 and the wave speed at $c=1$. The $\left(L^{\infty}, L^{2}\right)$ norm difference for the final versus the
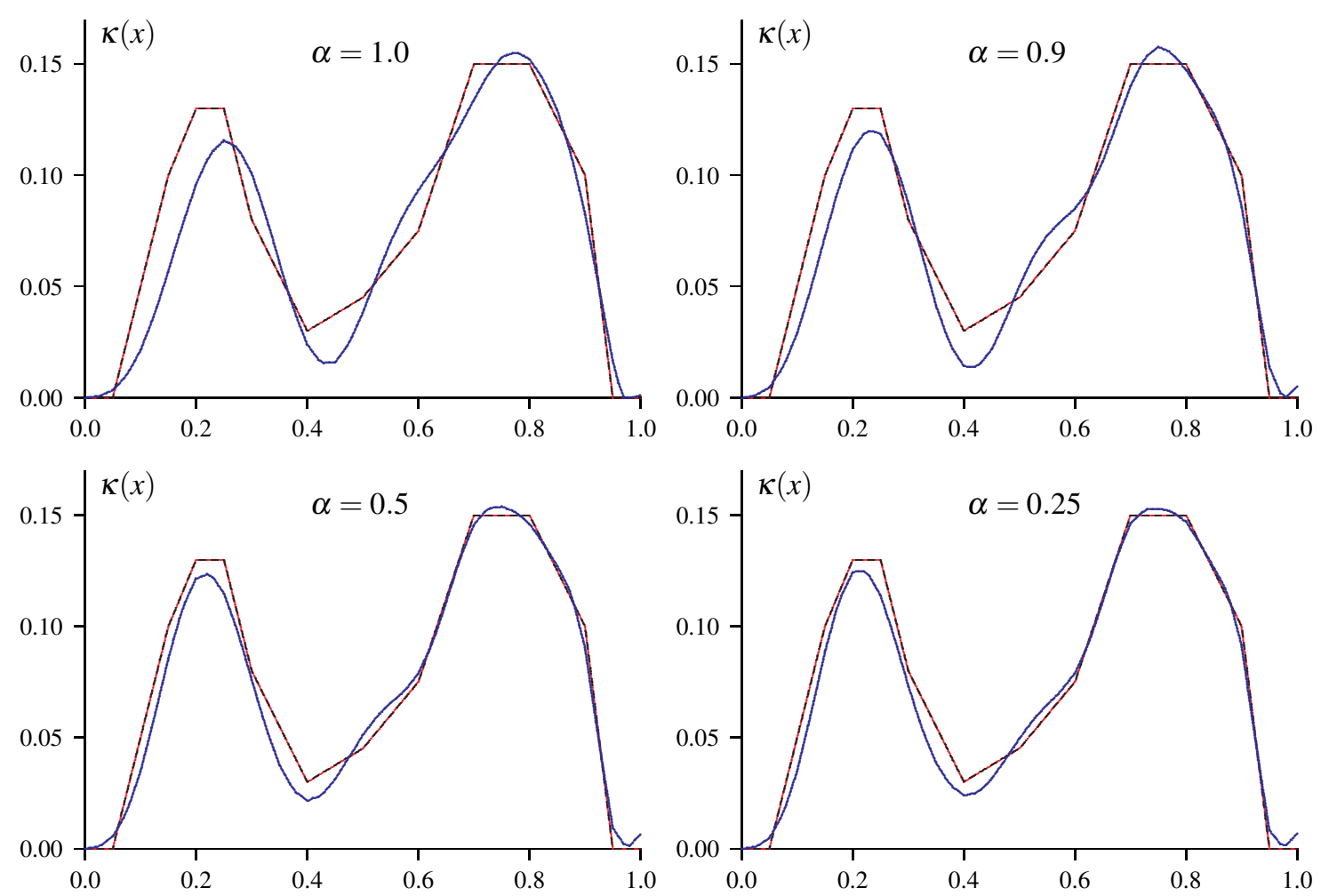

Figure 3: Reconstructions of $\kappa(x)$ for various $\alpha$ values. $\quad$ Noise $=\mathbf{0 . 1 \%}$

actual reconstruction were: $(0.109,0.078),(0.116,0.084),(0.184,0.126),(0.315,0.191)$, respectively and show the increase in resolution possible with a decrease in $\alpha$.

Note that the reconstructions of $\kappa$ are clearly superior at the right-hand endpoint due to imposed conditions as the wave is essentially transmitting information primarily from right to left but the amplitude is damped as it travels. The smaller the fractional damping the lesser is this effect which is also apparent from these figures.

The reconstructions naturally worsen with increasing noise levels as Figure 4 shows.

Figure 5 shows the singular values of the Jacobian matrix used in the (frozen) Newton method. Note that if the function $\kappa$ can be well represented by a small number of basis functions then the dependence with respect to $\alpha$ will be fairly weak. On the other hand, if a large number of basis functions are needed for $\kappa$ to be represented, then the dependence on 

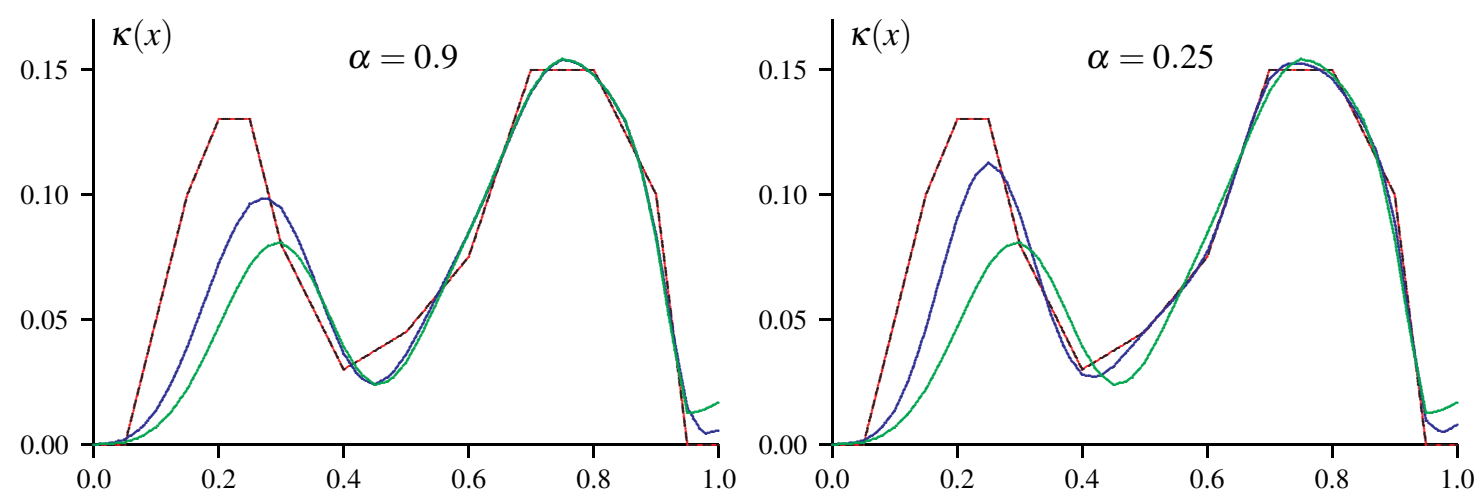

Figure 4: Reconstructions of $\kappa(x)$ for $\alpha=0.25,0.9 \quad$ Noise $=\mathbf{0 . 5 \%}$ (blue) and $1 \%$ (green)

$\alpha$ becomes much stronger although by this point the condition number of the Jacobian is already extremely high for all $\alpha$ and relatively few singular values are likely to be usable in any reconstruction with data subject to extremely small noise levels. The effect of damping
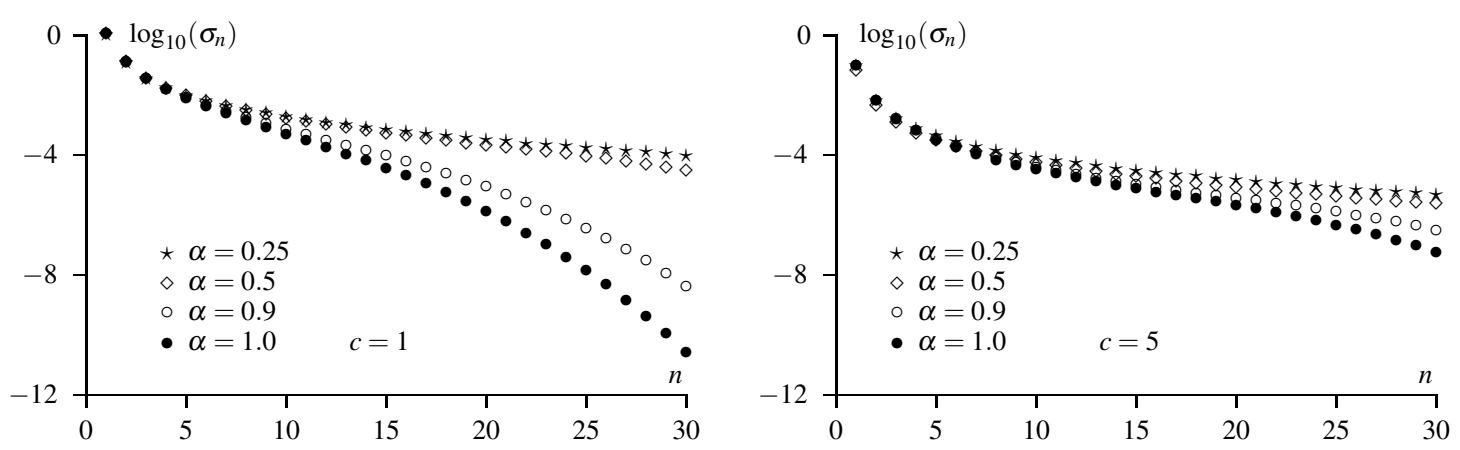

Figure 5: Singular values for various $\alpha$ values: left; $c=1$, right; $c=5$

is to directly contribute to the ill-conditioning and thus it is clear that for fixed $\alpha$ and $c$ this will increase as the coefficient $b$ increases. The degree of ill-conditioning as a function of the wave speed $c$ is less clear.

Figure 5 shows the singular values $\left\{\sigma_{n}\right\}$ of the Jacobian matrix for both $c=1$ and $c=5$. This illustrates the decay of the singular values and hence the level of ill-conditioning does depend on $c$ but certainly not uniformly for all values of the fractional exponent $\alpha$. For $\alpha$ near unity, that is damping approaches or is at the classical paradigm, there is a considerable increase in the smaller, high index singular values indicating the problem is much less ill-posed. for larger wave speeds $c$. For the smaller index $\sigma_{n}$ the ratio $\sigma_{n} / \sigma_{1}$ is almost the same indicating at most a weak effect due to the wave speed. Thus for a function $\kappa(x)$ requiring only a small number of basis functions the effect of wave speed is relatively minimal but this changes quite dramatically if a larger number of singular values are required. For $\alpha$ less than about one half the condition number $\sigma_{n} / \sigma_{1}$ becomes relatively independent of $c$-at least in the range indicated.

\section{Acknowledgment}

The work of the first author was supported by the Austrian Science Fund FWF under the grants P30054 and DOC78. 
The work of the second author was supported in part by the National Science Foundation through award DMS-1620138.

\section{References}

[1] A A Alikhanov. A Priori Estimates for Solutions of Boundary Value Problems for Fractional-Order Equations. Diff. Eq., 46(arXiv:1105.4592):660-666. 10 p, May 2011.

[2] L. Bjørnø. Characterization of biological media by means of their non-linearity. $U l$ trasonics, 24(5):254 - 259, 1986.

[3] V. Burov, I. Gurinovich, O. Rudenko, and E. Tagunov. Reconstruction of the spatial distribution of the nonlinearity parameter and sound velocity in acoustic nonlinear tomography. Acoustical Physics, 40:816-823, 111994.

[4] Wei Cai, Wen Chen, Jun Fang, and Sverre Holm. A Survey on Fractional Derivative Modeling of Power-Law Frequency-Dependent Viscous Dissipative and Scattering Attenuation in Acoustic Wave Propagation. Applied Mechanics Reviews, 70(3), 06 2018.

[5] Charles A. Cain. Ultrasonic reflection mode imaging of the nonlinear parameter B/A: I. a theoretical basis. The Journal of the Acoustical Society of America, 80(1):28-32, 1986.

[6] Michele Caputo. Linear models of dissipation whose $Q$ is almost frequency independent - II. Geophys. J. Int., 13(5):529-539, 1967.

[7] W. Chen and S. Holm. Fractional Laplacian time-space models for linear and nonlinear lossy media exhibiting arbitrary frequency power-law dependency. The Journal of the Acoustical Society of America, 115(4):1424-1430, 2004.

[8] Mkhitar M. Djrbashian. Integralnye preobrazovaniya i predstavleniya funktsiiv kompleksnoi oblasti. Izdat. "Nauka", Moscow, 1966.

[9] Mkhitar M. Djrbashian. Harmonic Analysis and Boundary Value Problems in the Complex Domain. Birkhäuser, Basel, 1993.

[10] Alan Edelman and H. Murakami. Polynomial roots from companion matrix eigenvalues. Math. Comp., 64(210):763-776, 1995.

[11] D. S. Grebenkov and B.-T. Nguyen. Geometrical structure of Laplacian eigenfunctions. SIAM Rev., 55(4):601-667, 2013.

[12] Sverre Holm and Sven Peter Näsholm. A causal and fractional all-frequency wave equation for lossy media. The Journal of the Acoustical Society of America, 130(4):2195-2202, 2011.

[13] Nobuyuki Ichida, Takuso Sato, and Melvin Linzer. Imaging the nonlinear ultrasonic parameter of a medium. Ultrasonic Imaging, 5(4):295-299, 1983. PMID: 6686896.

[14] Bangti Jin and William Rundell. A tutorial on inverse problems for anomalous diffusion processes. Inverse Problems, 31(3):035003, 40, 2015. 
[15] B. Kaltenbacher. Mathematics of Nonlinear Acoustics. Evolution Equations and Control Theory (EECT), 4:447-491, 2015.

[16] Barbara Kaltenbacher and William Rundell. On the identification of the nonlinearity parameter in the Westervelt equation from boundary measurements. Inverse Problems and Imaging, 2021. to appear.

[17] Barbara Kaltenbacher and William Rundell. Some inverse problems for wave equations with fractional derivative attenuation. Inverse Problems, 2021.

[18] F. Mainardi. Fractional Calculus and Waves in Linear Viscoelasticity: An Introduction to Mathematical Models. Imperial College Press, 2010.

[19] Francesco Mainardi and Rudolf Gorenflo. On Mittag-Leffler-type functions in fractional evolution processes. J. Comput. Appl. Math., 118(1-2):283-299, 2000. Higher transcendental functions and their applications.

[20] Kenichi Sakamoto and Masahiro Yamamoto. Initial value/boundary value problems for fractional diffusion-wave equations and applications to some inverse problems. $J$. Math. Anal. Appl., 382(1):426-447, 2011.

[21] Stefan G. Samko, Anatoly A. Kilbas, and Oleg I. Marichev. Fractional Integrals and Derivatives. Gordon and Breach Science Publishers, Yverdon, 1993.

[22] Thomas L. Szabo. Time domain wave equations for lossy media obeying a frequency power law. The Journal of the Acoustical Society of America, 96(1):491-500, 1994.

[23] Bradley E. Treeby and B. T. Cox. Modeling power law absorption and dispersion for acoustic propagation using the fractional Laplacian. The Journal of the Acoustical Society of America, 127(5):2741-2748, 2010.

[24] François Varray, Olivier Basset, Piero Tortoli, and Christian Cachard. Extensions of nonlinear B/A parameter imaging methods for echo mode. IEEE transactions on ultrasonics, ferroelectrics, and frequency control, 58:1232-44, 062011.

[25] Margaret G. Wismer. Finite element analysis of broadband acoustic pulses through inhomogenous media with power law attenuation. The Journal of the Acoustical Society of America, 120(6):3493-3502, 2006.

[26] Dong Zhang, Xi Chen, and Xiu-fen Gong. Acoustic nonlinearity parameter tomography for biological tissues via parametric array from a circular piston source-theoretical analysis and computer simulations. The Journal of the Acoustical Society of America, 109(3):1219-1225, 2001.

[27] Dong Zhang, Xiufen Gong, and Shigong Ye. Acoustic nonlinearity parameter tomography for biological specimens via measurements of the second harmonics. The Journal of the Acoustical Society of America, 99(4):2397-2402, 1996. 